Forthcoming in Behavioral and Brain Sciences

To be published with 20-40 commentaries and our response to these

https://www.cambridge.org/core/journals/behavioral-and-brain-sciences

\title{
The Number Sense Represents (Rational) Numbers
}

\author{
Sam Clarke \\ Department of Philosophy \& \\ Centre for Vision Research \\ S448 Ross Building, York University \\ Toronto, ON M3J 1P3 \\ spclarke@yorku.ca \\ http://www.sampclarke.net
}

\author{
Jacob Beck \\ Department of Philosophy \& \\ Centre for Vision Research \\ S448 Ross Building, York University \\ Toronto, ON M3J 1P3 \\ jbeck@yorku.ca \\ http://www.jacobbeck.org
}

\begin{abstract}
On a now orthodox view, humans and many other animals possess a "number sense," or approximate number system (ANS), that represents number. Recently, this orthodox view has been subject to numerous critiques that question whether the ANS genuinely represents number. We distinguish three lines of critique - the arguments from congruency, confounds, and imprecisionand show that none succeed. We then provide positive reasons to think that the ANS genuinely represents numbers, and not just non-numerical confounds or exotic substitutes for number, such as "numerosities" or "quanticals," as critics propose. In so doing, we raise a neglected question: numbers of what kind? Proponents of the orthodox view have been remarkably coy on this issue. But this is unsatisfactory since the predictions of the orthodox view, including the situations in which the ANS is expected to succeed or fail, turn on the kind(s) of number being represented. In response, we propose that the ANS represents not only natural numbers (e.g. 7), but also nonnatural rational numbers (e.g. 3.5). It does not represent irrational numbers (e.g. $\sqrt{ } 2$ ), however, and thereby fails to represent the real numbers more generally. This distances our proposal from existing conjectures, refines our understanding of the ANS, and paves the way for future research.
\end{abstract}

Keywords: analog magnitude system; approximate number system; number sense; numerical cognition; numerosity

\section{Introduction}

On a now orthodox view, humans and many non-human animals possess a number sense, or approximate number system (ANS), that affords a primitive and prelinguistic capacity to represent number. This hypothesis is supported by a large, and ever-growing, body of experimental work. But at its core, the orthodox view is dogged by two significant challenges.

First, many deny that the ANS actually represents number. Instead, critics claim that the ANS merely represents non-numerical confounds (Gebuis et al. 2016; Leibovich et al. 2017) or exotic substitutes for number, such as "numerosities," “quanticals" (Núñez 2017), or "pure magnitudes" (Burge 2010). Consequently, a cloud of uncertainty hangs over the orthodox view. Perhaps there is no number sense after all?

Second, even if the ANS does represent number, a question remains: what kind(s) of number does it represent? Proponents of the orthodox view have been remarkably coy on this issue. The default 
hypothesis seems to be that the ANS represents natural numbers - that is, positive whole numbers (e.g. 7) - but this is rarely made explicit. An alternative is that the ANS represents real numbers, which include not only natural numbers, but also rational numbers (e.g. 1/2) and irrational numbers (e.g. $\sqrt{2}$ ) (Gallistel \& Gelman 2000). There are other options as well, such as the hypothesis that the ANS represents rational but not irrational numbers. Choosing among these is important since the predictions of the orthodox view, including the situations in which the ANS is expected to succeed or fail, turn on the kind(s) of number being represented. Thus, without a clear sense of what the ANS represents, the orthodox view remains vague and underspecified.

The present treatment addresses both challenges on behalf of the orthodox view. After introducing the ANS in more detail and issuing some clarifications (\$2), we distinguish and address three lines of critique that have motivated skepticism about the ANS and its capacity to represent numbercritiques which we label the arguments from congruency, confounds, and imprecision (\$S3-5). We then highlight positive reasons for thinking that the ANS literally represents numbers, of a sort familiar from the math class, rather than ersatz numbers (e.g. "numerosities") (\$6). In so doing, we raise the neglected question: numbers of what kind? In answer to this question (\$7), we marshal evidence that the ANS represents both natural numbers and non-natural rational numbers. At the same time, we argue that the ANS fails to represent irrational numbers, and thus fails to represent the real numbers more generally. This distances our proposal from existing conjectures, refines our understanding of the ANS, and paves the way for future research on this topic.

\section{The ANS}

The ANS is a primitive and prelinguistic numerical system that is marked by a distinctive performance profile. For while the ANS facilitates computations over (sometimes quite large) numerical quantities, its numerical discriminations are imprecise and conform to Weber's Law. As such, discrimination deteriorates as the ratio between two numbers approaches 1:1. Thus, 8 is easier to discriminate from 10 than 10 is from 12 even though the absolute difference in number is the same. Meanwhile, 10 is as easy to discriminate from 5 as from 20. In each case, discriminability is determined by the ratio of the two quantities.

This performance profile distinguishes the ANS from other psychological systems that facilitate primitive numerical computations. For example, it distinguishes the ANS from a "parallel individuation" or "small number" system which facilitates precise numerical discriminations, but only among sets involving 4 items or less (Feigenson et al. 2004; Margolis 2020; but see Cheyette \& Piantadosi 2020). For, unlike the parallel individuation system, the ANS's numerical discriminations are imprecise (they are ratio sensitive) and they are not limited to small sets of items (the system might recognize that 30 dots are more than 20 dots). Furthermore, it distinguishes the ANS from a "texture density system." This is a system which, unlike the ANS, enables organisms to discriminate sets that are too crowded to parse, and (thus) perceived as texture (Burr et al. 2019). Crucially, the texture density system's performance is not predicted by Weber's Law but by a psychophysically distinct square root law, revealing a marked difference in its signature limitations (Anobile et al. 2016; Cicchini et al. 2016; Zimmermann 2018).

\subsection{Empirical Motivations}

The postulation of an ANS is not uncontroversial. Various critics deny that the system exists or that it genuinely represents number. Instead, they maintain that the relevant mechanisms and processes simply track non-numerical magnitudes, like areas and densities, or recherché alternatives to number, 
like "numerosities," as opposed to numbers themselves. In Sections 3-5 we examine such critiques in detail. But before we do, it is useful to consider some of the evidence that has (rightly or wrongly) motivated positing an ANS in the first place. Our focus will be on landmark studies and experimental paradigms that put naive readers in a position to appreciate where critics of the ANS are coming from, and where their critiques go wrong. For more comprehensive recent surveys, see Anobile et al. (2016), Anobile et al. (2021); Gebuis et al. 2016, Nieder (2016; 2020), and Odic \& Starr (2018).

Infant studies. In the early 2000s, various studies yielded evidence that young human infants can reliably track the numerical properties of large sets, albeit imprecisely and in accord with Weber's Law. For example, $\mathrm{Xu}$ and Spelke (2000) habituated six-month-old infants to visual arrays containing either 16 or 8 dots. When habituated to an 8-dot array, infants recovered interest when presented with a 16- or 4-dot array, but not a 12-dot array. Meanwhile infants habituated to a 16-dot array dishabituated to a 32- or 8-dot array, but not a 24-dot array. Since confounding variables such as brightness, density, and dot size were controlled for (Figure 1a), these findings were interpreted as showing that six-monthold infants can reliably discriminate the approximate number of items in two sets provided they differ by a suitably large ratio (e.g. 1:2). Subsequent studies suggested that these discriminative capacities improve with development. For instance, nine-month-olds were found to reliably discriminate sets that differ by a ratio of just 2:3 (Lipton \& Spelke 2003). In each case, performance decreased as numerical ratio approached 1:1, irrespective of the sets' precise cardinal values.

a.

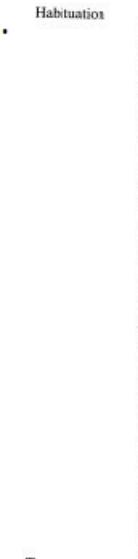

Test

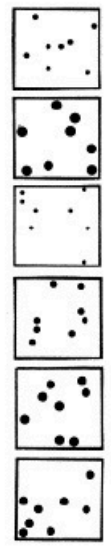

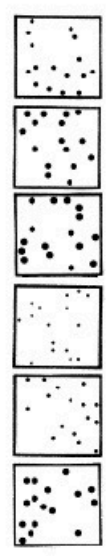

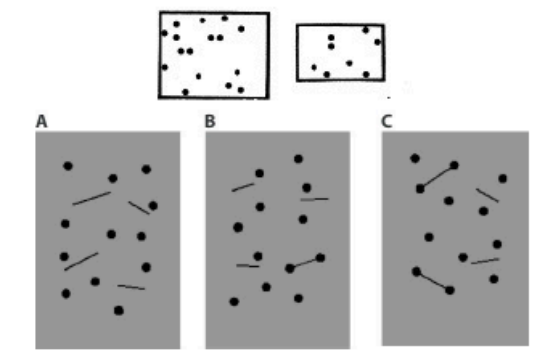

b.

O. Familiarization (2 min)

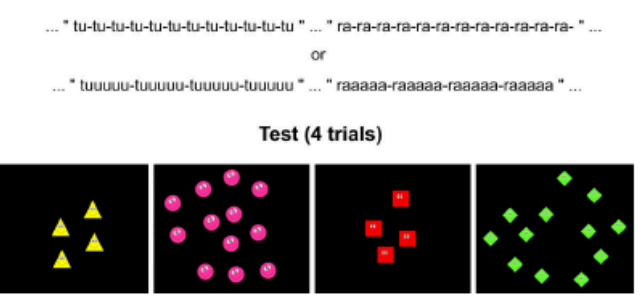

c.

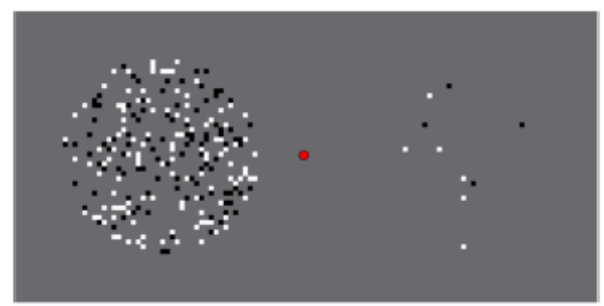

d.

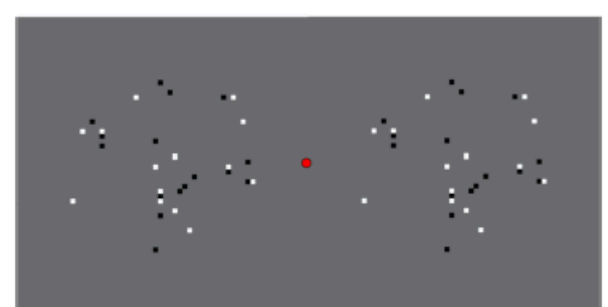

Figure 1a: Xu and Spelke habituated infants to arrays of dots and found that dishabituation would occur when test stimuli contained a number of dots that differed by a sufficiently large ratio. Since many confounds were equated in habituation displays (e.g. dot size) or test displays (e.g. density), this suggests a sensitivity to number itself. Reprinted from Xu \& Spelke (2000), p. B5, Figure 1, Copyright (C) 2000, with permission from Elsevier. Figure 1b: Izard et al. familiarized neonates to a number of heard tones before presenting them with visual arrays containing a number of items that either matched or failed to match the number of heard tones. Neonates looked longer at visual arrays containing a number of items that failed to match the number of tones they had been familiarized to. Reprinted from Izard et al. (2009), p. 10383, Figure 1, in line with PNAS's licensing agreement. Figure 1c. Burr and Ross instructed subjects to fixate on the dot in the upper 
figure of 1c for 30 seconds, at which point the lower figure from 1c was presented. Subjects reported that the display on the left appeared more numerous than the display on the right. Reprinted from Burr \& Ross (2008), Supplementary Data, Copyright (C) 2008, with permission from Cell Press. Figure 1d: He et al. showed that connecting dots with thin lines substantially decreased the number of dots arrays were estimated to contain. Reprinted from He et al. (2009), p. 510, Figure 1, Copyright (C) 2009, with permission from Springer Publishing Company.

Cross-modal infant studies. Cross-modal studies bolster the suggestion that these results reflect a genuine sensitivity to number. In one study, Izard et al. (2009) found neonates capable of matching numerical quantities across sets even when they were presented with a number of seen items and a number of heard sounds (Figure 1b). This complicates attempts to explain these findings in terms of a mere sensitivity to non-numerical confounds. After all, neonates in Izard et al.'s study could not have relied on (say) the size of seen items, or the total area of a seen set, when identifying a match, since properties of this sort could not have been heard. Nor could they have relied on the total volume or duration of heard stimuli, since properties of this sort could not have been seen. As such, these findings provide evidence that infants are able to abstract away from low-level confounds to track the numerical properties of sets they observe. And since controls indicated that these abilities are (again) ratio sensitive, they further implicate an ANS.

Pre-schooler studies. Of course, infants are notoriously difficult to study, requiring the use of indirect measures like looking time. But preschoolers can simply be asked which of two stimuli has "more" dots or tones, and since they are too young to reliably count, they appear to rely on an ANS when answering. In a striking illustration of this, Barth et al. (2005) showed that preschoolers could not only reliably answer which of two visual stimuli had "more" (e.g. red dots vs. blue dots), but that they were roughly as good at doing this across modalities (e.g. dots vs. tones) as within a single modality. This suggests that preschoolers' numerical competences are not tied to modality specific confounds. Once again, the numerical ratio between sets predicted performance, implicating an operational ANS in these children.

Adult studies. Studying adults adds a wrinkle to these investigations since most adults can use language to count, bypassing the ANS and its distinctive signature limitations. But when Barth et al. (2003) presented numerical stimuli too quickly for them to be explicitly counted, adults behaved like children. They discriminated stimuli in accord with Weber's Law in both intra- and inter-modal conditions. Similar results obtain during verbal shadowing tasks, where adults attempt to press a button a prespecified number of times while repeating a word, like 'the', to prevent them explicitly counting button presses. In studies of this sort, errors in the total number of button presses increase in proportion to the pre-specified number of target presses and are, therefore, predicted by Weber's Law (Cordes et al. 2001). So, again, these studies are indicative of a system with the performance profile of an ANS.

Adaptation studies. Burr and Ross (2008) adapted adult observers to a large or small number of dots by having them stare at a display for 30 seconds (Figure 1c). They then presented them with new displays of dots. Observers who had adapted to a large number of dots underestimated the number of dots on the new display, while observers who had adapted to a small number of dots overestimated the number of dots on the new display. Observers also reported consciously experiencing the aftereffect: subsequent displays appeared more or less numerous. Although some have argued that these effects involve adaptation to density rather than number (Durgin 2008; Dakin et al. 2011), recent studies control for density and other confounds (Fornaciai et al. 2016; DeSimone et al. 2020). Indeed, some work suggests that numerical adaptation effects occur between auditory and visual stimuli and are thus cross-modal (Arrighi et al. 2014). 
Dumbbell studies. In a different paradigm, subjects were presented with two visual arrays of items (squares or circles in Franconeri et al. 2009; dots in He et al. 2009) and thin lines. Their task was to say which array had more items, ignoring the lines. When the lines connected two items (effectively turning pairs of items into single dumbbell-shaped objects), subjects underestimated how many items were in the array (see Figure 1d). Franconeri et al. also showed that introducing a small break in the lines would substantially decrease this "dumbbell effect." Since displays with small breaks and displays of items connected with thin lines differ only slightly with respect to total surface area, spatial frequency, and other non-numerical magnitudes, these studies are (again) indicative of a genuine sensitivity to the number of objects observed.

Number neurons. Finally, all of these results can be linked to findings at the level of neural implementation. Neurons in the intraparietal sulcus (IPS) of monkeys respond selectively to specific numbers (Nieder 2016). Thus, specific neurons in the IPS respond preferentially when one perceives seven items. Interestingly, their response profile is noisy. Thus, neurons which are tuned to 7 often fire when one observes 6 or 8 items and occasionally when one observes 5 or 9 items. Indeed, noise levels increase with numerical quantity. This is what we might expect of neurons implementing an ANS since it seems to naturally explain why conformity to Weber's Law emerges at the behavioral level.

\subsection{Clarifications}

The preceding remarks do not provide a comprehensive overview of work on the ANS. Nor do they prove its existence. They simply provide readers with an initial sense of the vast, and seemingly convergent, evidence that has motivated positing an ANS in humans that represents number. The rest of this paper will be devoted to defending and extending this conjecture. But, before we proceed, it's important to clarify our aims.

Auxiliary claims. There are certain hypotheses that frequently accompany the postulation of an ANS which we can set aside for current purposes. These include hypotheses that the ANS is innate, that it is phylogenetically widespread, and that it is homologous across certain species. We can also remain largely neutral on the format of ANS representations (Beck 2015; 2018; Clarke forthcoming a), the informational resources it accesses in its computations (Mandelbaum 2013; Margolis \& Laurence 2008), and the details of its neural implementation (Lucero et al. 2020; Nieder 2016). Our question is simply whether the ANS represents numbers, and if so, numbers of what kind?

Referents vs. modes of presentation. We should distinguish the referents of ANS representations (what they represent) from their modes of presentation (how they represent). By analogy, the gustatory system plausibly represents $\mathrm{NaCl}$ concentrations. But it is a further question whether it represents $\mathrm{NaCl}$ concentrations as such - that is, as comprising molecules constituted by sodium and chloride atoms. Perhaps it is better to say that the gustatory system represents $\mathrm{NaCl}$ under the mode of presentation salty. We will argue that the ANS represents numbers (i.e., that numbers serve as the referents of the ANS), but under a unique mode of presentation that respects the imprecision inherent in the ANS (\$6). This will allow us to avoid a commitment to exotic entities such as "numerosities" without losing sight of the important differences between ANS representations and the precise numerical concepts that emerge later in development.

Perceiving numbers. Some may wonder how the ANS could refer to numbers given that numbers are abstract objects, not located in space or time. This worry might seem especially acute given that the ANS often operates perceptually (as in the adaptation studies introduced above). For how could anyone perceive the number seven? To clarify, note that philosophers standardly take perception to have 
an object-property structure. Perceptual states refer to objects, which are concrete particulars, and attribute properties to them. To perceive an object as red and square is to attribute the (abstract) properties of redness and squareness to a spatiotemporally located object. Among other virtues, this allows us to say what different perceptual states have in common. For example, the perception of a red apple and the perception of a red barn door concern different particulars but are alike insofar as both attribute redness. On this standard picture, abstract objects enter perceptual content through the attribution of properties, not through reference to objects. Likewise, if the ANS refers to numbers, it does so by enabling numbers to enter into contents via property attribution, not as objects of perception. So, your ANS might enable you to perceive the collection of apples on the table as being seven in number, but it wouldn't enable you to perceive the number seven itself-on its own, as it were-as an object.

Direct vs. indirect models of the ANS. Consider two distinct approaches to modeling the ANS. On a direct approach, the ANS fulfils its numerical function by individuating entities in the world and then counting these up. For instance, Dehaene and Changeux (1993) propose that the ANS performs an initial process of "normalization," which identifies individual items independently of confounding variables, like size and density. A later "accumulator" stage of processing then sums these, such that number is estimated. By contrast, an indirect approach eschews the initial individuation of items in favor of heuristic processes that derive number from continuous magnitudes. Thus, Dakin et al. (2011) and Morgan et al. (2014) propose that numerical quantity is estimated in visual perception on the basis of spatial frequency information at high and low bandwidths and is recovered in one step from information about area and density.

Although we believe that various phenomena, such as the dumbbell effects and cross-modal comparisons discussed in \$2.1, implicate a direct model of ANS processing (Anobile et al. 2016), at least in part, we do not consider this issue settled. It is thus important to stress that our defense of an ANS with genuine number content does not presuppose a direct model. This bears emphasizing because critics sometimes assume that if indirect models of the ANS are correct, then the ANS could represent nothing more than the continuous magnitudes (e.g. areas and densities) on which its computations would be based. This is a mistake. Perceived depth is computed on the basis of cues like binocular disparity, motion parallax, and retinal accommodation. But, of course, the visual system represents depth, and not (just) these cues. Correspondingly, the ANS might function to represent number even if its numerical estimations are based on a diverse range of continuous magnitudes and not on a representation of the individuals it functions to enumerate (Halberda 2019). To suppose otherwise is to confuse what the system is doing (e.g., functioning to track and represent numbers the computational level description with which we are concerned) for a specific account of how it does this (an algorithmic level description (Marr 1982)).

With these clarifications in view, we will now address three critiques which have targeted the ANS's alleged capacity to represent number ( $\$ \$ 3-5)$, offer positive reasons to think the system does represent number (\$6), and consider the specific kinds of number that it represents (\$7).

\section{The Argument from Congruency}

One reason critics doubt the existence of an ANS, with numerical content, derives from numerical congruency effects, where numerical judgments are influenced by the perception of irrelevant magnitude types. For instance, when subjects compare Arabic numerals and decide which picks out a larger number, their reaction times are influenced by font size. So, when the larger numeral is printed in a larger font (a "congruent" trial), they answer more quickly than when numerals are identical in size (a "neutral" trial). And when the smaller numeral has a larger font (an "incongruent" trial), they are 
slower and less accurate (Henik \& Tzelgov 1982; Gebuis et al. 2009; Gebuis et al. 2010). Similar effects occur in non-symbolic tasks (Figure 2). Thus, subjects tasked with determining whether one dot display is more or less numerous than another are influenced in comparable ways by things like average dot diameter, density, convex hull (the smallest convex area enclosing all the dots), and brightness (Cohen et al. 2006; Dakin et al. 2011; Gebuis \& Reynvoet 2012a; 2012b; Leibovich \& Henik 2014).

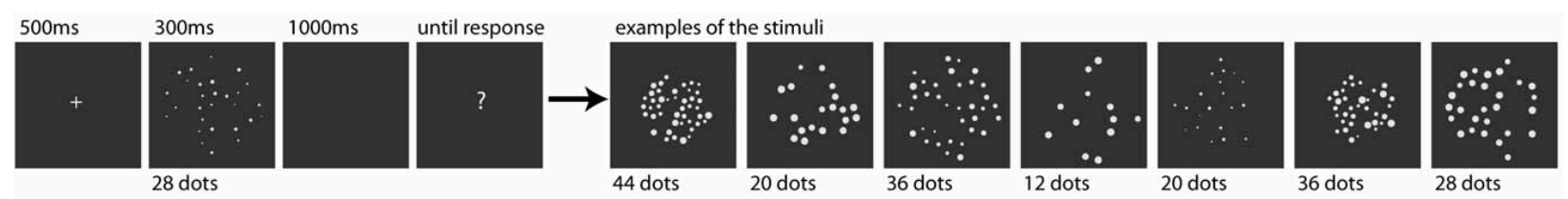

Figure 2. After fixation, subjects saw a display of dots briefly flashed on a screen. When the question mark appeared, they had to estimate the number of dots flashed by typing a number on their keyboard. The displays to the right of the arrow show examples of the stimuli that were used in this estimation task. Gebuis \& Reynvoet found that numerical estimations were strongly influenced by non-numerical confounds, like convex hull (a large convex hull disposed subjects to overestimate the total number of dots). Reprinted from Gebuis \& Reynvoet (2012c), p. e37426, Figure 1, in line with PLoS ONE's adopted CC BY License.

The argument from congruency proposes that these effects undermine the existence of an ANS that genuinely represents number (Leibovich et al. 2017; Gebuis et al. 2016). For if there were an ANS which represents number, we would expect relevant numerical judgments to be based entirely on its outputs. Thus, irrelevant non-numerical magnitudes should be ignored. But the existence of congruency effects suggests that (often) they're not. Or as Gebuis et al. (2016, p. 22) put it: If relevant numerical judgments are influenced by the perception of non-numerical magnitudes, then

why would there be an ANS system that can extract "pure numerosity"? What would be the use of having a system that can tell us exactly which cue [sic] at the passport control contains less people when it in the end adjusts this accurate answer in a possibly incorrect answer [sid] when for instance the length of the people in the cue [sic] is taken into account?

From the perspective of optimal design, Gebuis et al. propose that it makes little sense for a dedicated ANS to exist if its outputs are influenced by confounding variables in this way.

This objection presupposes that the ANS isn't governed by an indirect model that makes use of nonnumerical cues such as convex hull and length. For if it were, the ANS would obviously be influenced by those cues. But perhaps this is a safe assumption. Standard indirect models do not appeal to these cues, and nor, of course, do direct models.

Still, the argument faces other difficulties. An initial problem is that it overgeneralizes. It's well known that congruency effects affect judgements of uncontroversially perceptible magnitudes. For instance, judgments of duration exhibit congruency effects on size (Xuan et al. 2007; Lourenco \& Longo 2010), luminance (Xuan et al. 2007), length (Casasanto \& Boroditsky 2008), and distance (Sarrazin et al. 2004). So, if congruency effects demonstrate that numerical quantities are not represented by the ANS, then by parity of reasoning they would demonstrate that paradigmatically perceptible magnitudes (such as duration and distance) are not perceptually represented either.

To compound matters, congruency effects tend to be symmetric. For while numerical judgments are influenced by area and density, judgments of area and density are likewise influenced by number. Indeed, number often influences judgments of area and density at least as much as vice-versa (Cicchini et al. 2016; Savelkouls \& Cordes 2020; but see Yousif \& Keil 2020). So, if the fact that numerical judgments are influenced by area and density shows that number is not represented by an ANS, there should be equal or greater evidence that area and density are not represented either. In this way, the 
argument from congruency leads to an implausible skepticism about the perceptual representation of magnitudes quite generally.

These considerations indicate that the argument from congruency fails, but where does it go wrong? The argument errs in assuming that congruency effects are even in tension with the existence of an ANS, as we understand it. If there is an ANS which serves to represent numerical quantities, the observation of continuous magnitudes might introduce biases at the initial encoding stage, influencing the inputs the ANS receives, or at decision/response stages, altering outputs of the system. For example, at the response stage, congruency effects might reflect a Stroop-like byproduct of competition for a single response. In classic versions of the Stroop task, color and semantic processing compete for a single behavioral response (Johnson 2004). Numerical and non-numerical magnitudes might compete for a behavioral response in similar ways (see: Picon et al. 2019).

Alternatively, at the decision stage, congruency effects might reflect a useful strategy. For given Weber's Law, the ANS is not perfectly precise. To counteract that imprecision, subjects might make use of correlations among numerical and non-numerical magnitudes, perhaps in a Bayes' optimal way (Petzchner et al. 2015; see also: Martin et al. 2017). As Content et al. (2017, p. 20) note, "Continuous dimensions are indeed most often correlated with number in our experience of the world. No wonder that we would tend to use them, when possible, in comparing collections."

Gebuis et al. (2016, p. 22) object that while "the majority of studies show that larger sensory cues cause an overestimation [in number] and smaller sensory cues an underestimation, exceptions exist." For instance, Gebuis and Reynvoet (2012a) found that total surface area was sometimes inversely correlated with numerical estimation. This is something Gebuis et al. (2016) consider an embarrassment for proponents of the ANS who try to explain away congruency effects in terms of biases, since biases should (allegedly) be the same on all occasions. But we are not sure how puzzling this really is for proponents of the ANS. A Bayes' optimal inference might be expected to treat different magnitudes differently, and to even treat the same magnitudes differently in different contexts (the same premise does not always license the same conclusion!). But, even if this were not so, such considerations only appear more puzzling for skeptics of the ANS. The fact remains that numerical judgments are generally reliable in tasks of the sort under consideration. But it is not clear how this could be if those number judgments simply result from a sensitivity to non-numerical magnitudes (as skeptics maintain) and these are only insufficiently correlated with the numerical quantities in question. For example, if total surface area is sometimes positively, and other times negatively, correlated with numerical judgments, it is doubtful that numerical judgments (which are generally reliable) could simply be grounded in a sensitivity to total surface area.

\section{The Argument from Confounds}

The argument from congruency is unpersuasive. However, a more pressing objection concerns the fact that number cannot be presented to subjects independently of confounding variables. For instance, a visual display containing nine dots also contains dots with an average diameter, cumulative area, convex hull, and density. Similar points apply to heard or felt sets. Consequently, there has always been the worry that number isn't represented in studies of the above sort, only confounding variables. The argument from confounds claims that experimental attempts to evince an ANS with genuine numerical content are thereby undermined (Leibovich and Henik 2013; Leibovich et al. 2017; Gebuis et al. 2016).

There are actually two readings of this argument. On a strong reading, it's deemed impossible to adjudicate between the hypothesis that subjects represent numerical quantities in addition to various 
non-numerical confounds and the hypothesis that they merely represent these confounds. By contrast, a weak reading of the objection holds that while it may not be impossible to distinguish these hypotheses, it's sufficiently difficult that there is currently no empirical justification to favor the number sense hypothesis over a leaner alternative.

We see no reason to accept the argument in its stronger incarnation. Theories in science are always underdetermined by the data, and the selection of one theory over another often requires an inference to the best explanation (Duhem 1914). So, in psychology, there may never be a single experiment that eliminates all potential confounds. This does not rule out a science of the mind, however. It simply invites us to consider multiple studies in tandem and to ask whether these better support one hypothesis over viable alternatives. The postulation of an ANS with number content is unexceptional in this regard. For while number is an abstract property that cannot be observed in isolation (Halberda 2019), the same is true of many properties which are plausibly represented by the pre-linguistic mind, including causation (Kominsky \& Carey 2018) and agency (Gergely \& Csibra 2003). In each case, these hypotheses can be assessed against plausible alternatives. For to the extent that alternatives are undermined in controlled experiments, researchers can legitimately increase their credence in the relevant conjectures.

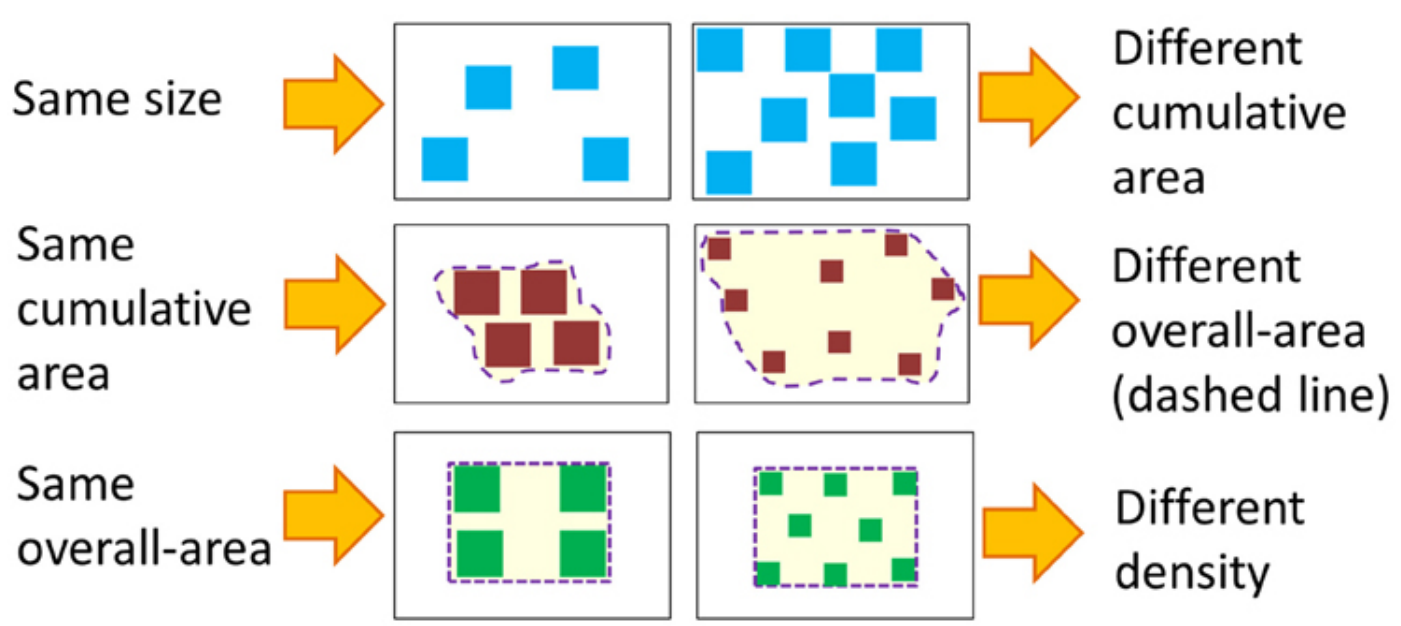

Figure 3: Attempts to control for one non-numerical confound often leave other confounds uncontrolled for. For example (top row), if two displays differ in number and the size of each item is constant, then the more numerous display must have a larger cumulative area. Reprinted from Leibovich and Henik (2013) p. 2, Figure 1, in line with Frontier's CC BY License.

This leaves the weaker reading of the objection. To appreciate its force, consider studies that examine our visual perception of number by presenting arrays of dots on a screen. Some such studies choose one potential confound - say, total surface area - and keep it constant while number varies. Critics like Leibovich et al. (2017, p. 4) correctly observe that this always leaves other confounds uncontrolled for (Figure 3). For example, if total surface area is kept constant while number increases, average dotsize will need to decrease (see DeWind et al. 2015 for a precise characterization of these tradeoffs). Consequently, finding that subjects discriminate a difference fails to establish whether they are tracking number or average dot-size.

Other studies vary non-numerical magnitudes across trials, such that no one confound correlates with number throughout the whole experiment. Thus, half the trials might keep total surface area constant while the other half keep average dot-size constant (Dehaene et al. 2005; Halberda et al. 2008). 
Alternatively, each of a range of non-numerical magnitudes might be varied across trials such that, throughout the experiment, they are congruent on half of the trials and incongruent on the other half (Barth et al. 2005; Nys and Content 2012). But while these controls suggest that subjects do not rely on a single confounding magnitude, Gebuis et al. (2016, pp. 23-24) and Leibovich et al. (2017, pp. 46) object that subjects could still be switching between cues throughout the experiment or relying on multiple confounds. For instance, when total surface area is held constant or made incongruent with number, they might rely on average dot-size; and when average dot-size is held constant or made incongruent with number, they might rely on total surface area. In this way, subjects might behave as if they are sensitive to number when they are only sensitive to non-numerical confounds.

We accept that this is conceivable, but we deny that it provides reasonable grounds for doubt. This is because a plausible skepticism about the ANS cannot be ad hoc. It cannot rest on a piecemeal strategy of finding one set of confounds to account for behavior in one trial, a second set of confounds to account for behavior in another trial, and so on. What is needed is a positive proposal that explains how some function of confounds, or some principled strategy for switching between these, accounts for what appears to be number-tracking behavior across a wide range of trials and experiments. Skeptics of an ANS fail to provide one. Instead, they simply observe that numerical judgments are influenced by non-numerical magnitudes - i.e., that they are subject to congruency effects. But, as we saw in $\$ 3$, these effects are fully compatible with the existence of an ANS that represents number.

To compound matters, experimental findings undermine the objection. Take the dumbbell effect discussed in \$2.1. As we explained then, connecting seen items with a thin line substantially reduces judgments of their number, while introducing a small break in these lines substantially reduces the effect (Franconeri et al. 2009; He et al. 2009; Kirjakovski \& Matsumoto 2016; see also: Fornaciai \& Park 2018). Given that displays with and without a small break are nearly identical with respect to non-numerical confounds, these studies suggest that number is tracked and represented, and that performance is not simply a function of tracking non-numerical confounds as critics allege. We have found no discussion of these studies by those who remain skeptical of the ANS and its capacity to represent number.

Additionally, some studies reveal that the relevant sensitivity to number differs markedly from our sensitivity to non-numerical magnitudes. Indeed, DeWind et al. (2015) compared how number, size, and spacing of dots affect relevant numerical judgments and found that judgments were more sensitive to number than to size or spacing. This suggests that number itself is represented. (For a reply, see Leibovich et al. 2017, p. 10, and for a rebuttal to this see Park \& Brannon 2017 and Tomlinson et al. 2020). Similarly, Cicchini et al. (2016) had subjects judge the area, density, and number of dots in visual displays, and found that number judgments are more sensitive than area and density judgments. Again, this suggests that subjects do not simply represent area and density, but also numerical quantity (see also Odic 2018; Savelkouls \& Cordes 2020).

Against this, it has been suggested that when perceived area is distinguished from mathematical area, number estimation is no longer shown to be more sensitive (Yousif \& Keil 2020). However, even here, the authors agree that "number estimation cannot be fully explained by perceived area" and that "the human visual system is certainly able to extract number." They only question whether number is being directly computed - an issue which is orthogonal to present concerns (see $\$ 2.2$ ).

Finally, we have already seen that cross-modal studies naturally eliminate potential confounds. For as was discussed in $\$ 2.1$, a static array of seen dots and a sequence of heard tones lack properties in 
common that could serve as a plausible crutch on which to base numerical comparisons. For while dots have a cumulative area, average diameter, and convex hull, tones have none of these properties. Since numerous studies demonstrate success in cross-modal tasks (Izard et al. 2009; Barth et al. 2003; Barth et al. 2005; Arrighi et al. 2014), this (again) undermines the argument from confounds.

Skeptics of the ANS do recognize this latter point. For instance, Leibovich et al. (2017) note that cross-modal studies provide "[a] very strong line of evidence supporting the ANS" (p.5). But while they proceed to question whether cross-modal studies on infants show that the ANS is innate, we can bracket these worries since we aren't focusing on the issue of innateness. For our purposes, a more relevant response comes from Gebuis et al. (2016). They acknowledge the existence of number studies in human adults demonstrating cross-modal adaptation (Arrighi et al. 2014) and cross-modal comparison (Barth et al. 2003; Tokita \& Ishiguchi 2012). They also acknowledge that these bear the hallmark of ANS-based-comparisons. In spite of this, they claim that such studies "do not present a clear result" (p.27). They reason that if number were represented amodally, there should be no cost to cross-modal comparisons. But while the existence of some such cost remains under dispute (contrast: Barth et al. 2003; Gebuis et al. 2016; Tokita et al. 2013), its discovery should not alarm proponents of an ANS. In intra-modal tasks, numerical comparisons are likely facilitated by confounding (typically, congruent) magnitudes (see \$3). Meanwhile, inter-modal tasks leave little opportunity for facilitation - after all, a static array of seen dots and a sequence of heard tones will lack properties in common that could serve to inform or bias numerical comparisons. In any case, it is the fact that cross-modal numerical comparisons are successfully executed at all that speaks in favor of an ANS.

In reply to all of this evidence, it might be argued that if indirect models of ANS computation are correct, then there has to be some description of what the ANS is doing that only appeals to nonnumerical magnitudes. So, given that we want to remain agnostic as between direct and indirect models (see \$2.2), what justifies us in holding that the ANS represents number rather than the mishmash of non-numerical magnitudes that would feature in that (hypothetical) description?

Earlier, we drew an analogy with depth, which is computed on the basis of diverse cues, such as binocular disparity, motion parallax, and accommodation. Even so, we said that people perceive depth, and not just these cues. It's important to appreciate why we said that. The reason is that representation of depth offers a unifying explanation that the motley of cues cannot. On one occasion the visual system might rely primarily on binocular disparity to compute the depth of an object; on another occasion it might rely primarily on motion parallax; on a third occasion it might rely primarily on convergence; and so on. Appealing to a common representational kind in each case (representation of depth) allows us to provide a coherent, unified account of one's ability to judge depths in these and other circumstances, and it supports largely accurate predictions regarding the judgments you will make about depths on the basis of what you perceive. In short, it supports generalizations. It also helps explain why you are sensitive to these cues in the first place; that is, it helps explain what function they are serving. The hypothesis that the ANS represents number enjoys the same advantages over the hypothesis that the system merely represents a mishmash of non-numerical magnitudes to which a hypothetically adequate indirect model would appeal.

\section{The Argument from Imprecision}

This brings us to what is perhaps the most prominent critique of the ANS's capacity to represent number: the argument from imprecision. While this argument has a more philosophical flavor than the preceding objections, it has been repeated many times, often by scientists. We'll begin by outlining and critiquing a generic version of the argument, before examining two specific incarnations of the 
argument, due to Carey (2009) and Núñez (2017). Once again, we'll suggest that, as things stand, the argument fails to undermine the hypothesis that the ANS represents number.

\subsection{The Generic Version}

The argument from imprecision begins from the observation that the ANS is imprecise. When it processes a collection of 23 entities, say, it does not reliably produce a representation of exactly 23 . If it did, we would have no difficulty discriminating collections of 23 from collections of 22. But, given Weber's Law, we know that discrimination suffers as the ratio between magnitudes approaches 1:1. Thus, ANS representations must be imprecise in some way or another. But numbers themselves are not imprecise. 23 is exactly one more than 22 and exactly one less than 24.23 even differs from 23.00000000000001 by a precise amount (exactly .00000000000001). So (the argument goes), the ANS fails to represent numbers.

As stated, this argument is invalid. From the premise that the ANS represents imprecisely and the premise that numbers are precise it simply doesn't follow that the ANS fails to represent numbers. To illustrate, consider that non-numerical magnitudes such as distance, duration, and weight are also precise. There is a fact of the matter about exactly how heavy a given person is, and a fact of the matter about how much heavier/lighter one person is than another. But while non-numerical magnitudes, like weight, can be represented precisely, they needn't be. You can represent Jones as "240 pounds" or "as heavy as an NFL linebacker." In either case you are representing Jones' weight; what differs is how precisely you are doing so. Prima facie, the same is true of number. You can represent the number of coins in your pocket precisely as "exactly six" or imprecisely as "approximately six", or "several" (cf. Ball 2017, p. 126).

Why, then, do so many theorists find the argument from imprecision compelling? The answer, we suspect, lies in a tacit commitment to the sensitivity principle. In broad strokes, the sensitivity principle holds that representing an entity requires sensitivity to its essential properties. But this principle can be read in a number of ways.

On a strong reading, it might be fleshed out as follows.

The Strong Sensitivity Principle: if X has properties $\mathrm{p}_{1} \ldots \mathrm{p}_{\mathrm{n}}$ essentially, then representing X requires being sensitive to all of $\mathrm{p}_{1} \ldots \mathrm{p}_{\mathrm{n}}$.

If true, this would render the above argument valid. For if we were to grant that numbers are essentially precise, acceptance of this strong sensitivity principle would license the conclusion that a capacity to represent numbers requires sensitivity to this precision. Since this is something that the ANS does not provide, acceptance of the principle could thereby license the conclusion that the ANS fails to represent number.

The trouble is: this strong version of the sensitivity principle is false. To illustrate, notice that while water is essentially $\mathrm{H}_{2} \mathrm{O}$, representing water does not require sensitivity to the property of being composed from two hydrogen atoms and an oxygen atom. You can think about how much you'd like a glass of water without knowing anything about the chemical composition of the water you desire. Likewise, gold is essentially the element with atomic number 79, but thinking that you'd like a gold watch does not require any sensitivity to atomic numbers. In fact, you needn't even be sensitive to the difference between gold and fool's gold. Many people who are ignorant of chemistry and metallurgy nevertheless think about water and gold (Putnam 1975; Burge 1982). 
A similar point applies to perception. It seems to be an essential property of continuous magnitudes that they are dense; that between any two distances, durations, or weights, there is always a third. But while perception represents these magnitudes, it has a limited resolution that prevents it being sensitive to their denseness. In representing motion, for example, the visual system relies on Reichardt detectors that cannot distinguish continuous from discrete changes in position (Green, 2018). That's why a string of Christmas lights that turn on in succession give rise to an illusion of motion (the phi phenomenon).

For this reason, proponents of the argument from imprecision must weaken their reading of the sensitivity principle. To this end, they might propose some version of the following.

The Weak Sensitivity Principle: if X has properties $\mathrm{p}_{1} \ldots \mathrm{p}_{\mathrm{n}}$ essentially, then representing X requires being sensitive to some of $\mathrm{p}_{1} \ldots \mathrm{p}_{\mathrm{n}}$.

This weakened sensitivity principle enjoys prima facie plausibility. After all, it is perhaps plausible to suppose that the capacity to represent $\mathrm{X}$ requires sensitivity to some of X's essential properties even if it doesn't require sensitivity to all of these. For what else could make it the case that $\mathrm{X}$ is being represented rather than some other entity, Y?

The trouble is, even if this is granted, the weak sensitivity principle fails to fix the argument from imprecision. For even if representing numbers requires sensitivity to some essential properties of numbers, it doesn't follow that representing numbers requires sensitivity to the specific property of being precise. As a result, proponents of the argument from imprecision still require an (as yet unstated) reason for thinking that sensitivity to numerical precision is necessary to represent number.

Can a convincing reason be provided? While we can't rule it out, we have a hard time envisioning what it would look like. ${ }^{1}$ Nor do we find any hints in the writing of those advocating the argument from imprecision. To illustrate, we will now consider two prominent incarnations of the argument, before turning to some positive reasons for thinking that the ANS represents numbers.

\subsection{Carey on Imprecision}

Carey (2009, p.295) invokes two versions of the argument from imprecision to maintain that the ANS is "not powerful enough to represent the natural numbers." While these arguments have prima facie appeal —in fact, one of us endorsed them in earlier work (Beck 2015) — they are unsound.

\footnotetext{
${ }^{1}$ One possibility would be to maintain that representing X requires being sensitive to those properties that feature in X's definition, or "individuation conditions." Thus, if it is definitive of numbers that they are precise, then representing numbers would require sensitivity to their precision (cf. Peacocke 2020, pp.154-156). But that approach requires a highly controversial commitment to definitions (Quine 1951; Fodor 1998), which proponents of the argument from imprecision do not defend. Moreover, the most plausible versions of this principle are restricted to conceptual representation under a canonical mode of presentation (Peacocke 2020). (To canonically represent X, one must be sensitive to the properties that are definitive of X.) But we are primarily concerned with specifying the referents of ANS representations, not their modes of presentation. Thus, while we maintain that the ANS represents numbers, we are happy to concede that it does so noncanonically and non-conceptually.

2 Although Carey denies that the ANS represents natural numbers, she occasionally says it represents cardinal numbers (e.g. Carey 2009, p.136). As Burge (2010, p.480, n.82) observes, these claims are hard to square. (The natural numbers are often identified with the finite cardinals.) Ball $(2017$, p. 135, n.9) attempts to resolve the tension, proposing that while natural numbers are best defined in terms of the successor relation, cardinal numbers are best defined in terms of Hume's principle (that the Fs and Gs are equinumerous if and only if there is a one-to-one correspondence between them). Thus (according to Ball), whereas representing natural numbers requires sensitivity to the successor relation, representing cardinal numbers requires sensitivity to Hume's principle. Carey may then maintain that the ANS represents cardinal
} 
First, Carey contends that ANS representations "fail to capture small numerical differences between large sets of objects" (ibid., p.294). For example, she writes that "the difference between eight and nine is not experienced at all" because eight and nine "cannot be discriminated" (ibid., p.295). But as Halberda (2016) points out, this erroneously presupposes that discriminability is binary. In fact, discriminability decreases smoothly as ratio increases, so there is no simple cut-off after which differences are not discriminated. In theory, even 999 and 1000 may be discriminated above chance given enough trials.

Furthermore, Carey assumes that if subjects aren't sufficiently sensitive to small differences between natural numbers (e.g., eight vs. nine), then they cannot represent natural numbers. But short of presupposing the problematically strong version of the sensitivity principle, it's unclear what justifies this assumption. The visual system represents distances even though it's relatively insensitive to small differences between these (e.g., 80 vs. 81 centimeters). This is because, there is nothing problematic in the thought that a precise distance (e.g., 80 centimeters) might be represented imprecisely. The same holds for number. To suggest otherwise is to mistake what the system represents (e.g., integers) for how it represents this (e.g., precisely or imprecisely).

Carey's second argument is that since the ANS treats five and six as more similar than four and five, it "obscure[s] the successor function," and thus cannot represent natural numbers (Carey 2009, p.295). Here, the suggestion is that a capacity to represent natural numbers requires a sensitivity to the successor function because the successor function is essential to natural numbers. But, again, this argument seems to presuppose the strong sensitivity principle, which should be rejected. For, short of providing some (as yet unspecified) reason for thinking that a sensitivity to the successor function is necesssary to represent numbers, it fails to follow that a system (like the ANS), which is insensitive to this, fails to represent number, even if it is granted that the successor function is an essential feature of number.

\subsection{Nüñez on Imprecision}

Núñez (2017) introduces his version of the argument from imprecision by noting that the ANS's numerical discriminations are "rarely exact" (p.417) - they conform to Weber's Law. But as he sees it:

A basic competence involving, say, the number 'eight', should require that the quantity is treated as being categorically different from 'seven', and not merely treated as often - or highly likely to be - different from it. (ibid.)

Thus, Núñez proposes that the ascription of genuine numerical content to an ANS would require that it quantify "in an exact and discrete manner" lest this amount to nothing more than "loose" talk (p.418). Since this is something that the ANS does not do, Núñez proposes that the ANS does not represent numerical quantities at all.

To be clear, Núñez is not proposing that the ANS is an approximate number system which represents numerical quantities inexactly. He is denying that it produces any numerical content whatsoever. This is evident in his "crucial distinction" between cognition that is "numerical" and cognition that is merely "quantical" (a term Núñez invents). Among other things, "quantical" cognition denotes "quantity-related capacities" that do not meet the requisite level of precision to qualify as genuinely

numbers but not natural numbers if she maintains that the ANS is sensitive to Hume's principle but not the successor relation. We deny that such sensitivity is required in either case. But we also don't see the justification for claiming that the ANS is sensitive to Hume's principle. Thus, we think the tension Burge identifies in Carey's exposition remains. 
numerical. Thus, Núñez urges that unless a system meets the requisite level of precision, it would be inappropriate to suppose it represents anything more than non-numerical quantities.

In saying this, Núñez lumps the ANS's representations in with perceptual representations of other magnitudes, such as duration, brightness, distance, and chemical concentrations. All of them are on a par. They are all "quantical." But notice that, in making this suggestion, Núñez fails to explain why a capacity to represent number requires a sensitivity to their precise nature. In this way, Núñez fails to address the challenge posed to proponents of the argument from imprecision in $\$ 5.1$.

On a charitable reading, it might be acknowledged that an appeal to "quantical" dimensions does, at least, offer an alternative to the view that the ANS represents numbers. Thus, it may seem attractive to those who are antecedently skeptical of the ANS's capacity to represent number. For this reason, it's important to stress that this is an unhelpful way of understanding matters. For one, an appeal to "quanticals" obscures a crucial distinction in this context. This is because, numerical quantities are higher order in that they can only be assigned relative to a sortal - a criterion for individuating the entities being counted (Frege 1884). If we want to determine how many shoes are in your closet, it's not enough to open your closet and round up your shoes. We also need to decide whether we're counting individual shoes, pairs of shoes, or types of shoes. By contrast, if we want to know how much the shoes in your closet weigh, or what their total volume is, there is nothing further we need to do once we've identified the set of shoes. As Burge (2010, p.472) notes, numerical quantities thus have a "second-order character" that non-numerical quantities lack. As such, it's important to recognize that the ANS represents properties with this second-order character even though its representations are imprecise.
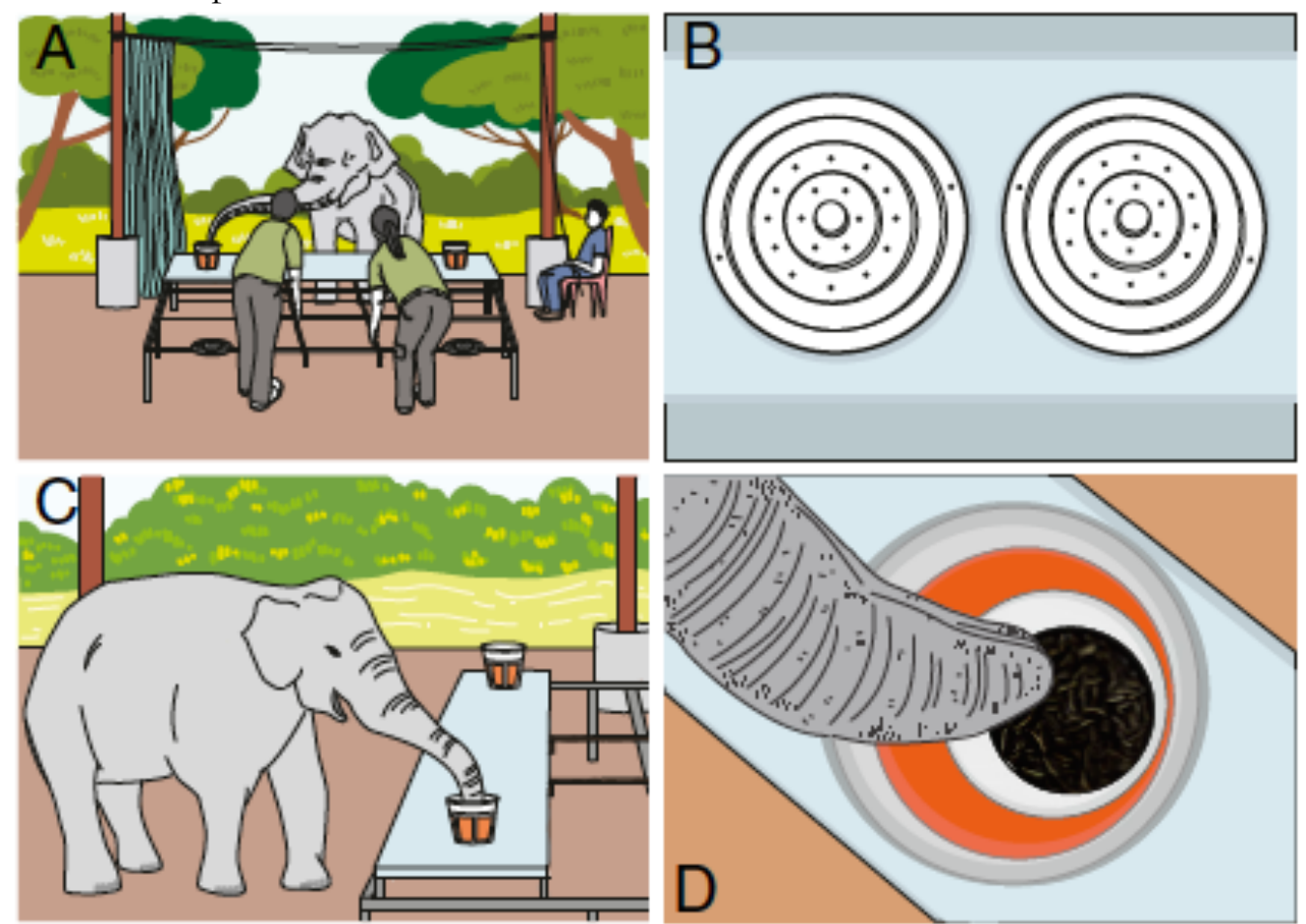

Figure 4. Elephants smelled two buckets containing sunflower seeds (A) with opaque, perforated lids (B). After they made their selection $(\mathrm{C})$, the lid was removed, and they could eat the seeds (D). Elephants generally chose the bucket with a greater number of seeds, which (presumably) also had the more intense odor. Illustration by Nuttayapond Doungcharoen. Reprinted from Plotnik et al.'s (2019), p. 12567, Figure 1, in line with PNAS's licensing agreement. 
To illustrate, recall the dumbbell studies by He et al. (2009) and Franconeri et al. (2009). When items are connected by thin lines, they are judged to be less numerous than when lines do not connect the items or contain small breaks. This indicates that the ANS takes a stand on how the entities in the array are individuated when they are being enumerated. Thus, it is not merely estimating the size of a first-order quantity. Rather, the ANS is sensitive to the second-order character of number. Since this second-order character is essential to number, it follows that the weak sensitivity principle is satisfied. There is at least one essential property of number to which the ANS is sensitive.

Appealing to the "quantical" obscures this feature of ANS representation since even first-order quantities qualify as "quantical" in Núñez's sense. To see why this should matter, consider a recent study by Plotnik et al. (2019). Here, elephants were presented with pairs of buckets containing sunflower seeds. These had opaque, perforated lids, allowing elephants to smell, but not see, their contents. Plotnik et al. found that elephants would preferentially select the bucket containing a greater quantity of sunflower seeds, albeit imprecisely and in accord with Weber's Law (Figure 4). On this basis, they took their results to corroborate studies indicating the existence of an ANS in these creatures (e.g., Irie et al. 2019). But note, while this might be so, it neglects a simpler possibility: elephants were simply sensitive to the intensity of the odor emanating from the buckets, leading them to approach the bucket with the stronger odor (and hence more seeds). On this account, Plotnik et al.'s findings would be orthogonal to the presence or absence of an ANS with genuine numerical content; they would simply demonstrate these creatures' formidable capacity for olfaction. ${ }^{3}$

In studies with humans, non-numerical confounds have been carefully controlled for (see $\left.\int 4\right)$. By contrast, Plotnik et al. fail to distinguish between the hypothesis that elephants represent number and the hypothesis that they represent odor. But it's a substantive question which is correct. And it's a question we would wish to answer whether or not the relevant discriminations are imprecise. This is because there's a basic distinction between representing a first-order magnitude, like odor, and an ability to abstract away from this to represent a higher-order numerical magnitude. Núñez's approach obscures this important distinction because even odor representations are imprecise and quantitative, and thus satisfy his criteria for being "quantical."

\section{Number vs. Numerosity}

We have now considered three arguments which purport to undermine the conjecture that the number sense represents number and found no reason to reject this conjecture. We have also uncovered considerations that seem to support it. For instance, we have seen that ANS representations track second-order properties of concrete pluralities (albeit imprecisely) as opposed to first-order quantities. It should also be noted that ANS representations enter into arithmetic computations such as greaterthan and less-than comparisons, addition, subtraction, multiplication, and division (Barth et al. 2005; Barth et al. 2006; Barth et al. 2009; McCrink \& Spelke 2010; McCrink \& Spelke 2016; McCrink et al. 2012). Pending a convincing argument to the contrary, these considerations indicate that the ANS literally represents some kind of numerical magnitude.

Even so, it's crucial not to lose sight of the limitations inherent in the ANS that make its representations so different from the precise number concepts most human adults have at their

\footnotetext{
${ }^{3}$ Our worry here is very different from that which underpins the argument from confounds. Our worry is that Plotnik et al. failed to control for a single, specific capacity that we have independent reason to attribute to elephants. By contrast, the worry that motivates the argument from confounds is that there could be some unspecified and gerrymandered mix of cues that experimenters fail to control for.
} 
disposal. There is a conceptual change that occurs when children acquire the capacity to count and properly use number words in their native language (Carey 2009). There is also a deficit in human adults who retain an ANS but do not have access to precise number concepts - e.g., because their community is bereft of natural number terms (Gordon 2005; Pica et al. 2005), or because they suffer damage to their prefrontal cortex (Lemer et al. 2003).

It is vital to acknowledge and mark this difference. But we should distinguish two ways of doing so. According to the first approach, these two types of representation differ because they represent different things. Whereas precise number concepts represent literal numbers (e.g., positive integers), imprecise ANS representations merely represent "numerosities," which are a kind of ersatz number. According to the second approach, these two types of representation differ not in what they represent - both literally represent numbers of a sort familiar from the math class - but in how they do so. That is, they have different modes of presentation. In the remainder of this section, we'll argue that this second approach is preferable.

It is notable that, throughout the ANS literature, the term "number" is assiduously avoided in favor of the neologism "numerosity." We suspect that this reflects an assumption that, strictly speaking, the ANS represents numerosities, not numbers. But what is a numerosity? Despite widespread employment of the term, ANS researchers almost never say. Apart from Núñez's appeal to "quantical" dimensions, which we criticized above, we know of only one other proposal that clearly distances numerosities from numbers: Burge's (2010) conjecture that these are "pure magnitudes."

Burge is skeptical that the ANS represents numbers but finds himself frustrated by psychologists' woolly use of the term "numerosity," calling it "a hedge term used to apply to number-like properties" (Burge 2010, p.472). To gain clarity, Burge recommends drawing inspiration from the ancient Greek theory of magnitudes attributed to Eudoxus and reported by Euclid.

According to Eudoxus, magnitudes divide into two species, continuous and discrete. Continuous magnitudes include length, weight, and duration. Discrete magnitudes include natural numbers. In addition, Eudoxus recognized the genus of these two species, pure magnitudes, which he used to account for (what we would now call) irrational numbers, such as $\sqrt{2}$. The Pythagoreans had noticed that the diagonal of a square cannot be expressed as the ratio of two whole numbers. Eudoxus' theory of pure magnitudes was intended to provide a way to express such quantities. Burge speculates that the ANS represents pure magnitudes, which support basic arithmetic operations, but do not differentiate between discreteness and continuity. He writes, "I conjecture that the early Greeks articulated and formalized basic animal and childhood capacities when they theorized about magnitudes and ratios in a way that is unspecific as to whether the magnitudes are numbers or continuous quantities" (Burge 2010, p.483). ${ }^{4}$

\footnotetext{
${ }^{4}$ Buijsman (2021) endorses Burge's suggestion, and supplements it with an account of indeterminate vehicles to explain the ANS's imprecision. Buijsman (2021, p. 310) acknowledges that readers might wonder why he says that the ANS represents pure magnitudes rather than natural numbers, and replies that natural numbers "cannot be indeterminate" because, "There are no alternative choices for ' 1 ' as the unit value of the natural numbers which are equally good, whereas there are alternative choices for ' $1 \mathrm{~cm}$ ' which are equally good, namely 1 inch, 1 meter, and so on." We doubt that 1 is the only "equally good" unit of measurement for natural numbers. (Is it a shanda to buy bagels by the dozen?) But even if it is, and even if individual natural numbers cannot be indeterminate, ranges of natural numbers can be. So, we still don't see why indeterminacy favors the hypothesis that the ANS represents pure magnitudes over the hypothesis that it represents (ranges of) numbers.
} 
The thing is, the ANS refers to a second-order property of collections (see \$5.2). It attributes a quantity relative to a way of sorting or individuating particulars. But as the genus of discrete and continuous magnitudes, pure magnitudes do not differentiate between magnitudes that have a second-order character (e.g. natural numbers) and magnitudes that lack such a second-order character (e.g. length). Pure magnitudes are thus poorly suited to capturing the contents of ANS representations.

But if numerosities aren't pure magnitudes then what are they? The simple fact is, no one has any idea. And that seems like good reason to avoid positing numerosities as the contents of ANS representations. Better to say that the ANS represents numbers of a familiar sort. ${ }^{5}$

In saying this, we avoid a curious double standard that plagues discussions of the ANS. For, as we have already noted, perceptual representations of non-numerical magnitudes, like distance, duration, and weight, are also imprecise and governed by Weber's Law. But we have not come across a single passage which concludes that we thereby represent "distancosity," "durationosity," or "weightosity." And for good reason. While no one knows what a distancosity, durationosity, or weightosity would be, distance, duration, and weight are respectable and familiar entities in our scientific ontology. The imprecision inherent in our discrimination of non-numerical magnitudes is thus not taken to prevent distance, duration, or weight from being represented; it's merely taken to modify the way in which they are represented. Taking the ANS to represent number rather than numerosity allows for greater consistency with our treatment of non-numerical magnitudes.

At this point, it would be nice if we could support our proposal with a naturalized theory of content that explains why the ANS represents number rather than numerosity. But we don't have one, and the leading theories on offer (e.g., Shea 2018; Neander 2017) aren't pitched at the right level of granularity. This being said, our search for the referent of a representation should be biased towards entities we have independent reason to posit in our scientifically informed ontology. Among other virtues, this allows psychological explanations invoking representational content to be integrated with explanations from other sciences, like biology. We are thus in agreement with Burge when he counsels that the visual system of the frog should be taken to represent flies rather than undetached fly parts because undetached fly parts "are not kinds that ground biological explanations of the frog's needs and activities" (Burge 2010, p. 322; see also p. 466). Since biological explanations appeal to numbers, not numerosities, we find reason to favor the conjecture that numbers are the referents of ANS representations, and not numerosities as others propose.

Finally, it bears emphasizing that there is no need to invoke peculiar entities such as numerosities to make sense of differences between ANS representations and precise number concepts. These differences can simply be captured by appeal to their modes of presentation. As Frege (1892) observed, two names can refer to the same object even though they differ in what we intuitively think

\footnotetext{
${ }^{5}$ A reviewer suggests that number researchers may be reticent to jettison the term "numerosity" because it's useful to refer to a concrete plurality - for example, a collection of six dots on a screen. But one can represent a concrete pluarlity without representing its numerical value, and what is distinctive of the ANS is not merely that it represents concrete pluralities, but that it attributes numerical values to them. Another reviewer wonders what researchers who are accustomed to the term "numerosity representation" should use instead to avoid confusion with precise number concepts. Where context isn't sufficient, we recommend "ANS representation," "approximate number representation," or "analog number representation." By contrast, the precise number concepts acquired later in development can simply be called "precise number concepts," "acquired number concepts," or "conceptual number representations."
} 
of as their meanings. Thus, while "Hesperus" and "Phosphorus" both refer to Venus, they have different modes of presentation. It was a substantive empirical discovery that the names co-refer. And what goes for names, goes for mental representations (Dummett 1981; Evans 1982; Peacocke 1992; Burge 2005). This is clearly true in thought-the Babylonians' concepts Hesperus and Phosphorus had distinct modes of presentation. Likewise, modes of presentation differ between perception and thought. As Peacocke (1986) noted, knowing the length of a piano in feet and inches may not settle whether it will fit along a wall in your living room even if you're looking straight at the wall. The reason is not that the property you entertain in thought differs from the property you entertain in perception, but that you entertain the property in different ways - that is, under different modes of presentation. This is also the most natural thing to say about how ANS representations differ from precise number concepts.

In fact, there is independent reason to think that ANS representations and precise number concepts differ substantially in their mode of presentation. That is because the representations involved have different formats. The fact that the ANS obeys Weber's Law indicates that its representations are analog, like a mercury thermometer (Beck 2015; 2018; 2019; Clarke forthcoming a). Precise number concepts, by contrast, have a non-analog, language-like format. Differences in format spawn differences in mode of presentation (Beck 2013) but are compatible with sameness of reference. Just as an analog watch and a digital watch both represent time, the ANS and our precise number concepts both represent number-albeit in different ways.

In saying this, we do not assume that precise number concepts are ontogenetically grounded in the ANS. For example, we do not claim that learners acquire precise number concepts by mapping them onto their ANS representations. In fact, there are reasons to be skeptical of this claim (Carey \& Barner 2020). Such reasons largely derive from the mismatch in format and precision between ANS representations and precise number concepts. But, as we've seen, that mismatch in format and precision does not require a mismatch of reference; on the contrary, it is better characterized by a mismatch in mode of presentation.

This is important to recognize because researchers sometimes suggest that the ANS can directly ground precise number concepts if, and only if, it represents numbers. For example:

if the ANS represents numbers, then its representations can directly link to our processing of symbolic numbers. If something like pure magnitudes... are represented, then the ANS has to bridge a gap to be relevant to symbolic number tasks. (Buijsman 2021, p. 304)

But this is a mistake. A gap needs to be bridged whether or not the ANS represents numbers.

Are we just playing a linguistic shell game? Is there a substantive difference between saying that the ANS represents numerosity (not number) and saying that it represents number but with a different mode of presentation than precise number concepts?

The difference is substantive. There is a clear difference between systems that represent distinct quantities, such as a clock and a thermometer, and systems that represent the same quantity in different ways, such as a digital clock and an analog clock. Researchers who take the ANS to represent nonnumerical or faux-numerical quantities are simply mischaracterizing the ANS. This has practical implications, such as a tendency to overlook the second-order character of ANS representations and to underestimate what it takes to tap the ANS itself (as in Plotnik et al. 2019). It also leads number researchers on a fool's errand-searching for, and attempting to illuminate, mysterious properties, like "quanticals." But if we are right that the ANS represents number, researchers can halt the wild goose 
chase and refocus more fruitfully on factors that contribute to the unique mode of presentation found in ANS representations, such as their format, imprecision, and computational role.

Strange as it is to have to say: the number sense represents number.

\section{What Kind(s) of Number?}

We have now argued that the ANS represents numbers of a familiar variety: extant arguments to the contrary are unpersuasive ( $\$ \$ 3-5)$ and there is positive reason to endorse the conjecture $(\$ 6)$. But this raises the neglected question noted in $\$ 1$ : numbers of what kind?

Before proceeding, we should stress that there are various things one might be asking with this question. So, to clarify, we'll be asking how fine-grained the numbers represented by the ANS are. Thus, we'll be asking whether the ANS represents natural numbers, which are relatively coarse-grained (\$7.1), real numbers, which are extremely fine-grained (\$7.2), or rational numbers, which have an intermediate grain (\$7.3). In so doing, we'll remain neutral on whether the ANS is committed to a specific axiomatization, analysis, or ontology of the numbers it represents. We'll also remain agnostic about whether we should think of the ANS as representing precise numbers $(7,1.5$, etc.), precise numbers with a confidence estimation attached (Halberda 2016), numerical intervals (5-9, 1.25-1.75, etc.) (Ball 2017), or probability distributions over numerical intervals. While interesting and important, these questions are orthogonal to whether the ANS represents natural, real, or rational numbers. Since the question of whether the ANS represents natural, real, or rational numbers already bears on the computations an ANS performs and facilitates, answering this question would constitute a substantial contribution to our understanding of the ANS.

\subsection{Natural Numbers}

To begin, let's consider the conjecture that the ANS represents natural numbers, or positive integers. While rarely defended (but see Ball 2017), this conjecture seems to be assumed by those who maintain that precise integer concepts derive from the ANS (Wagner \& Johnson 2011; Dehaene 2011; Piazza 2011; Park \& Brannon 2013; Starr et al. 2013; Nieder 2017). Indeed, once one concedes that the ANS represents numbers, the conjecture that the ANS represents natural numbers may seem to constitute the default hypothesis. For on this view, the ANS functions to represent whole numbers, like 7 and

8 , and not comparatively esoteric numbers, such as $\sqrt{2}$, whose initial identification constituted major mathematical discoveries.

While we are skeptical that the ANS grounds precise integer concepts (Carey \& Barner, 2020), there is strong reason to think the ANS does represent positive integers. We say this because, as we have seen, the ANS functions to keep count of whole items, at least in paradigm cases. Exactly what these items might be varies from stimulus to stimulus, or even as a result of shifting attention. Thus, the ANS might represent the number of visual objects in an array, or objects of a certain type (such as closed shapes, or circles, or red circles as opposed to green ones, and so forth). Or rather than representing material objects, it might represent a number of events, such as rabbit jumps (Wood \& Spelke 2005), or auditory items in a sequence, such as tones or phonemes heard. In each case, the ANS functions to represent the number of whole items in the relevant set. Since the number of whole items in a given set is expressed by a positive integer, we conclude that, at a minimum, the ANS represents positive integers.

\subsection{Real Numbers}


In one of the few discussions to address the kinds of number the ANS represents, Gallistel and Gelman (2000) defend a striking hypothesis: the ANS goes beyond representing natural numbers by representing real numbers, which include not only integers, but also rational numbers, which can be expressed as a ratio of integers (e.g. 1.5), and irrational numbers, which cannot be so expressed (e.g. $\sqrt{2}$ ). In fact, Gallistel and Gelman conjecture that the ANS primarily represents irrational numbers, since "all but a negligible fraction of [the real numbers] are irrational" (p.59). This is a remarkable result. Is it correct?

If the ANS represents irrational numbers then, all things being equal, we would expect this to be manifested in ANS-governed behavior. But Gallistel and Gelman do not point to any behavioral evidence of this sort; nor do we know of any. For while there is ample evidence that the ANS represents whole numbers, we know of no evidence that it represents $\sqrt{2}$, say. As it stands, the hypothesis that the ANS represents irrational numbers would seem unsupported by existing evidence.

Why, then, do Gallistel and Gelman endorse the hypothesis? They reason as follows. First, since duration is a continuous magnitude, they infer that it cannot be represented by anything discrete. So, the neural magnitude that represents duration must be continuous. Next, drawing on evidence in rats (Church \& Meck 1983), they infer that numbers are represented by neural "magnitudes indistinguishable from those which represent duration" (Gallistel \& Gelman 2000, p.62). Thus, the ANS must also use a continuous neural magnitude. Finally, since real numbers are continuous, but integers are not, they conclude that the ANS must represent real numbers rather than just integers.

This argument is problematic on various fronts. Firstly, while duration is a continuous magnitude, it is unclear whether duration's continuity is reflected in the grain of duration representations. Just as there is a dearth of evidence that the ANS represents irrational numbers, such as $\sqrt{2}$, there is a dearth of evidence that durations such as $\sqrt{ } 2$ seconds are represented by comparable mechanisms. So, while duration representations may be very fine-grained, there is no evidence that they are genuinely continuous (Laurence \& Margolis 2005, p. 223, n.7). Gallistel and Gelman may be led to think otherwise because they take Weber's Law to be evidence of vehicles that are continuous neural magnitudes. But so long as the right type of noise is introduced, Weber's Law can be explained by discrete neural vehicles, such as the number of neurons firing above some threshold within a given population (Maley 2011; Beck 2015).

Secondly, Gallistel and Gelman's argument rests on the mistaken assumption that the content of a representation mirrors its format. Thus, they assume that if duration is represented as continuous, then the vehicle employed must be continuous too; and if the vehicles employed by the ANS are continuous, then the content of the ANS is a continuous number line (reflecting the real numbers). But as Laurence and Margolis (2005) point out in a compelling critique of Gallistel and Gelman's argument, we use discrete vehicles to represent continuous contents all the time. For example, discrete symbols like " $\pi$ " and " $\sqrt{2}$ " express precise irrational numbers. Conversely, digital computers use continuous magnitudes, such as voltage, to represent discrete values (Lewis 1971; von Neumann 1958). Thus, "There is nothing at all incoherent about mental magnitudes representing discrete values" (Laurence \& Margolis 2005, p. 224).

Given the shortcomings in Gallistel and Gelman's argument, and the general lack of empirical evidence for their conclusion, the hypothesis that the ANS represents irrational numbers should be rejected pending a convincing argument to the contrary. 


\subsection{Rational Numbers}

At this point, it may be tempting to suppose the ANS merely represents natural numbers. After all, there is compelling reason to think that the system does, in fact, represent natural numbers, and the most prominent account on which the ANS goes beyond representing these is unpersuasive. Against this, we recommend an intermediate position. On our view, the ANS goes beyond representing natural numbers (e.g. 7) by representing (non-natural) rational numbers (e.g. 3.5). That is because the ANS represents ratios among positive integers, in addition to positive integers themselves, and rational numbers are expressible as ratios among positive integers. While we do not take this hypothesis to be definitively established, we consider it well-motivated and strongly suggested by a range of empirical findings. Moving forward, it should be our working hypothesis.

The proposal we are making is closely related to a suggestion from the developmental and educational psychology literatures according to which there is a "ratio processing system" (RPS) which stands to our understanding of fractions in the way a number sense has traditionally been seen to stand to whole number understanding (Siegler et al. 2013; Matthews \& Chesney 2015; Lewis et al. 2016; Matthews et al. 2016; Bhatia et al. 2020; Binzak \& Hubbard 2020). That being said, it isn't always clear whether the RPS is supposed to be a component of the ANS (as we'll suggest) or a separate system. Moreover, the hypothesis that the RPS represents rational numbers is not always clearly distinguished from the conjecture that it represents real numbers more generally. Indeed, it is sometimes assumed that evidence for the former hypothesis would be a stepping-stone towards vindicating the latter conjecture (e.g., Matthews et al. 2016, p. 191).

Consequently, it is worth considering why one might expect a system like the ANS to represent rational numbers, and not just natural numbers. The reason, we suggest, is that organisms need to reason under uncertainty. They need to draw inferences (e.g., about the future) from limited data. The capacity to engage in fast and efficient probabilistic reasoning would, thus, be enormously advantageous (Tenenbaum et al. 2011). And in fact, a flood of research suggests that even human infants engage in probabilistic reasoning of this sort (Girotto \& Gonzalez 2008; Xu \& Garcia 2008; Xu \& Denison 2009; Gweon et al. 2010; Téglás et al. 2011; Denison \& Xu 2014; Kayhan et al. 2018). To do so, they need to represent probabilities; and the most straightforward way to do this is to represent rational numbers.

In one study to probe representations of this sort, McCrink and Wynn (2007) habituated 6-month-old infants to multiple examples of a single numerical ratio and found that they would subsequently look longer when presented with a novel ratio (Figure 5a). For instance, infants habituated to displays containing a 4:1 ratio of blue pellets to yellow Pacman shapes recovered interest when presented with a display containing a 2:1 ratio. Since the number of individual pellets and Pacman shapes was varied across habituation displays (while keeping the ratio between these constant and other confounds controlled for) this suggests that infants locked onto, and subsequently responded to, a change in the numerical ratio between these elements, abstracting away from the specific number of pellets and/or Pacman shapes presented in each display. Furthermore, just as Xu and Spelke (2000) found that 6month-old infants could reliably discriminate absolute numerical values in a 2:1 ratio but not a 3:2 ratio, McCrink and Wynn (2007) found that they could reliably discriminate the ratio 4:1 from 2:1 but not the ratio 3:1 from 2:1. This similarity in performance is suggestive of a shared system.

Using a different approach, Denison and Xu (2014) tested ratio understanding in 10- to 12-monthold infants (Figure 5b). They presented each infant with two lollipops, one pink and one black, to see 
which they preferred. They then showed the infants two transparent jars containing pink and black lollipops in different ratios and sampled from these populations by placing a lollipop from each jar in an opaque cup without allowing the infant to see its color. The infants reliably walked or crawled towards the sample that came from the jar with the higher ratio of their preferred lollipop. Crucially, this persisted even when the jar with the higher ratio of preferred lollipops contained fewer preferred lollipops in absolute terms. Thus, for pink-preferring infants, when one jar contained 16 pink and 4 black lollipops, and the other contained 24 pink and 96 black lollipops, they approached the sample drawn from the first jar rather than the second. (See also Xu \& Garcia 2008; Xu \& Denison 2009; Denison \& Xu 2010; Fontanari et al. 2014; and Kayhan et al. 2018. ${ }^{6}$
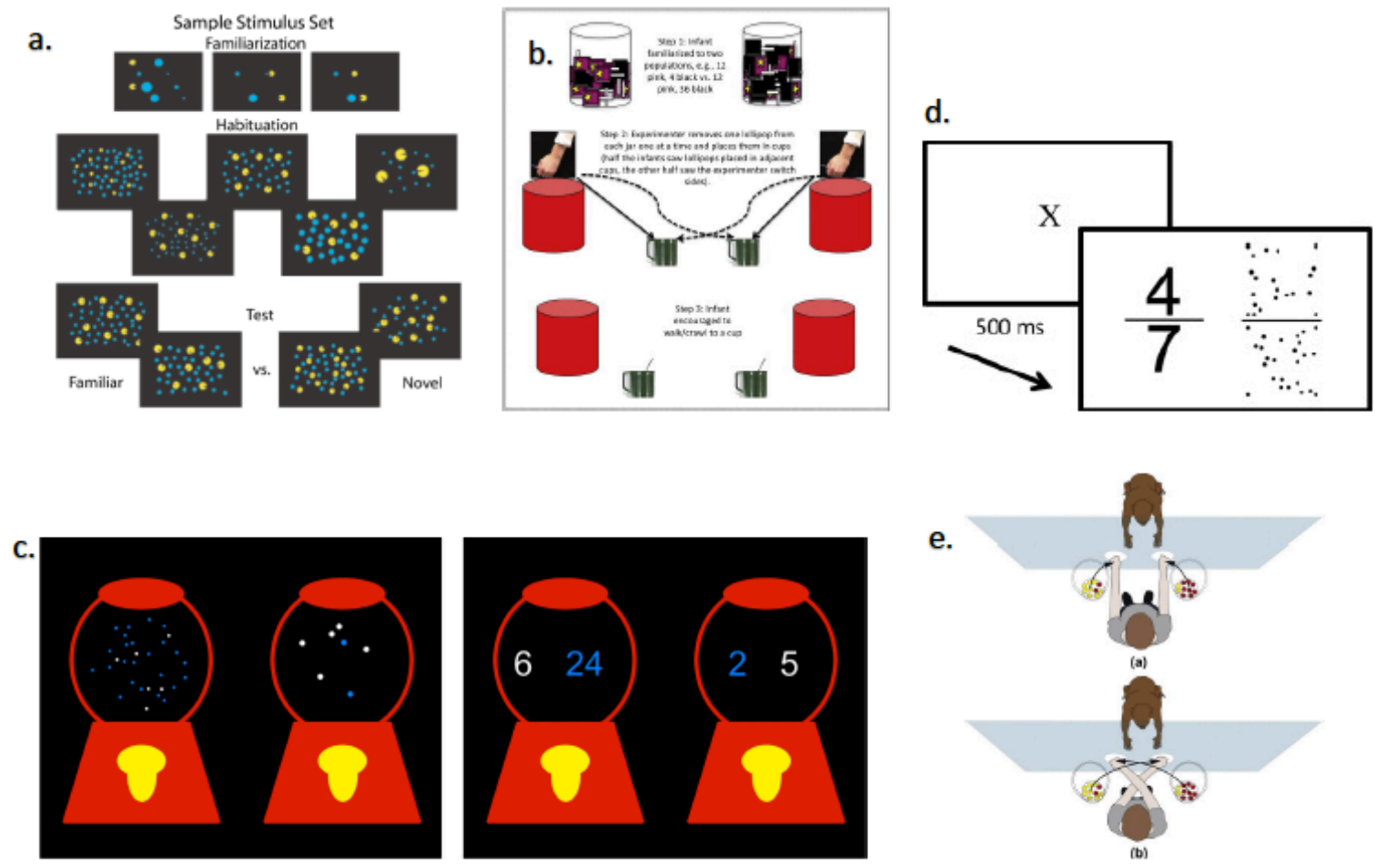

e.

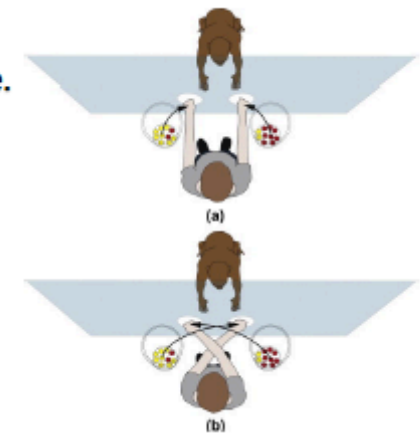

Figure $5 a$. The habituation stimuli in the figure illustrate the 4:1 ratio that infants were habituated to. Reprinted from McCrink and Wynn (2007), p. 742, Figure 1, Copyright (C) 2007, with permission from SAGE Publications. Figure 5b. The test condition, reprinted from Denison \& Xu (2014), p. 338, Figure 1, Copyright (C 2014, with permission from Elsevier. Figure 5c. Illustration of the non-symbolic (left) and symbolic (right) ratio comparison tests, reprinted from Szkudlarek \& Brannon (2021), p. 3, Figure 1, Copyright (C 2021, with permission from the Society for Research in Child Development. Figure 5d. Example of a symbolic and non-symbolic fraction comparison, reprinted from Matthews \& Chesney (2015), p. 33, Figure 1, Copyright (C) 2015, with permission from Elsevier. Figure 5e. Rakoczy et al.'s study mirror's Denison and Xu's (2014), but with non-human apes instead of infants. Reprinted from Rakoczy et al.'s (2014), p. 63, Figure 1, Copyright (C) 2014, with permission from Elsevier.

One limitation of this lollipop task is that infants could have succeeded by representing ratios of (pink vs. black) surface areas rather than numbers. But while Denison and Xu (2014) didn't control for area, McCrink and Wynn (2007) did. Studies with older children also control for area and other non-

\footnotetext{
${ }^{6}$ One issue that isn't settled by this study_or, to our knowledge, any other extant study — is whether infants succeed by using ratios or fractions. Do they choose the jar that contains 16 pink lollipops because the ratio 16 pink: 4 black is more favorable than the ratio 24 pink: 96 black? Or do they choose it because the ratio 16 pink: 20 total lollipops is more favorable than the ratio of 24 pink: 120 total lollipops? Only the latter ratios are equivalent to fractions-i.e., 4/5 vs. 1/5. Adding additional colors and then testing for a cost (in terms of errors or reaction time) might provide some insight. But either way, what is represented goes beyond mere integers, and thus seems to require an appeal to rational numbers.
} 
numerical quantities. For example, Szkudlarek and Brannon (2021) presented 6- to 8-year-olds with pairs of depicted gumball machines (Figure 5c). Each gumball machine was filled with either blue and white 'gumballs' (dots), or blue and white Arabic numerals specifying the number of blue and white gumballs contained therein. In each case, the children were tasked with selecting the gumball machine with the best chance of producing a gumball of a desired color, taking into account the ratio of blue to white gumballs contained therein. They were able to do so reliably in both non-symbolic (dot) and symbolic (numeral) conditions, even though children this age have not yet begun to study fractions in school and struggle with precise fraction comparisons. Since subsequent analyses indicated that the children's performance could not be attributed to simpler heuristics (e.g. choosing the machine with more desired gumballs, or the machine with fewer undesired gumballs), these results suggest that 6- 8year-olds represent numerical ratios. And, since this capacity was manifested in symbolic displays, where the numerator and denominator were specified with Arabic numerals, non-numerical confounds were largely eliminated.

Matthews and Chesney (2015) ran a related study in which college students were tasked with choosing the larger fraction when symbolic arrays (e.g. ${ }^{4} / 7$ ) were pitted against discrete non-symbolic arrays in which the numerator and denominator were expressed with dots, and again when these discrete nonsymbolic arrays were pitted against continuous non-symbolic arrays in which the numerator and denominator were each replaced by a circle of variable area (Figure $5 \mathrm{~d}$ ). Subjects succeeded in these comparisons even though they answered too quickly to have explicitly counted the dots in the discrete non-symbolic arrays. Importantly, their reaction times and errors were predicted by Weber's Law, suggesting that they used their ANS to represent ratios among whole numbers.

Finally, just as there is evidence that nonhuman animals discriminate among absolute numerical values, there is evidence that monkeys (Drucker et al. 2016; Tecwyn et al. 2017) and nonhuman apes (Rakoczy et al. 2014; Eckert et al. 2018) discriminate numerical ratios (Figure 5e).

Given converging evidence that the ANS supports comparisons among ratios of positive integers, we conclude that the hypothesis that the ANS represents rational numbers deserves to be provisionally endorsed. But notice two things. First, this conjecture is still pitched at a computational level of analysis (\$2.2). Thus, it is not wedded to a specific account of the system's underlying architecture. For this reason, it is a further question to what extent (if any) the neural and/or psychological mechanisms involved in the ANS's representation of natural and rational numbers overlap. To clarify, notice that the visual system is often viewed as unified by its function, despite comprising relatively autonomous sub-modules performing dedicated tasks at various levels of visual analysis (Clarke, forthcoming b; Fodor, 1983; Marr, 1982). Analogously, our suggestion that the ANS represents both natural and rational numbers allows us to remain neutral on the (important but additional) question of whether it comprises autonomous components dedicated to natural number representation, on the one hand, and rational number representation (the RPS proper), on the other. This is because, even if these components are distinct, and even largely encapsulated from one another, the ANS (as we have understood it) remains unified on account of its unified functional profile: representing numbers in accord with Weber's Law.

Second, our conjecture that the ANS represents rational numbers does not commit us to claiming that the system represents every rational number or even most rational numbers. This bears emphasizing because the conjecture has previously been dismissed on these grounds. For instance, Marshall (2017, p.49) claims that the ANS cannot represent the rational numbers because the rational numbers are dense - between any two rational numbers there is always a third - and the ANS does not respect this 
feature. For while the ANS probably represents 2.5 and 2.75 , there is no evidence that the ANS can represent 2.7452294861. This objection should, however, have a familiar ring: it seems to presuppose the strong sensitivity principle, which (as we've seen) must be rejected. Just because rational numbers are essentially dense doesn't mean that the ANS must be sensitive to their denseness to represent them. Just as the ANS can represent natural numbers like 7 even though it cannot represent all of the natural numbers (e.g., one trillion is surely beyond its upper limit), the ANS can represent positive rational numbers such as 2.5 even if it cannot represent all positive rational numbers.

Earlier we argued that the ANS represents numbers, not non-numerical magnitudes, because it is sensitive to the second-order character of numbers, which is an essential property of numbers but not non-numerical magnitudes. Now we're arguing that it represents rational numbers, not just integers, because it's also sensitive to ratios, which are an essential property of rational numbers but not integers. Notice, however, that there are no essential properties of irrational numbers, but not rational numbers, to which the ANS is sensitive (so far as we can tell). Thus, there is no parallel reason to say that the ANS represents real numbers more generally. The train terminates at rational numbers.

If the ANS can represent rational numbers, what would prevent it from representing irrationals? We suspect that this may be a byproduct of how it operates, privileging the representation of whole entities. After all, it is plausible that all of the aforementioned studies involved the ANS, first, representing natural numbers of concrete pluralities, and only then deriving ratios (hence, rational numbers) therefrom. For instance, in McCrink and Wynn's (2007) study it is natural to suppose that the system, first, represented natural numbers of pellets and Pacman shapes, and only then contrasted these so as to identify the ratio between them. But since there is no way to represent irrational numbers as a ratio of natural numbers, the existence of some such process could not undergird the representation of real numbers more generally.

Of course, much work remains to be done developing and testing our hypothesis. The study of ratio understanding is still relatively young. The aforementioned studies are vulnerable to defeater explanations - e.g., pitched in terms of a mere sensitivity to ratios between non-numerical confounds such as area. Thus, evidence that the ANS represents rational numbers remains provisional. But psychologists could adapt paradigms which discredit confound-based explanations for natural number tracking ( $(4)$, such as cross-modal comparisons and dumbbell stimuli, to adjudicate these concerns. It would also be nice to see studies that more directly test whether identical ratios (e.g., 4:8 and 16:32) are treated as such.

Our skepticism surrounding the ANS's ability to represent irrational numbers stems (in large part) from the lack of positive evidence to support this proposal. But considerations of parsimony to one side, absence of evidence is not evidence of absence. Thus, scientists might seek out empirical evidence that irrational numbers (e.g. $\pi$ ) feature in the computations the ANS performs. For instance, they might consider the ANS's potential involvement in calculating square roots or logarithms. Of course, care must be taken to ensure that the ANS is being tested and not some non-numerical magnitude system. For example, the ability to compute a circle's area from its radius would not require computations over any numbers, let alone irrational numbers, if non-numerical magnitudes such as length and area are represented in a unit-free manner (Peacocke 1986). Moreover, even if these computations did implicate numerical magnitudes, careful controls/arguments would be needed to show that they involve a representation of irrational numbers and not rational approximations thereof. 
Regardless, we believe that reflection on these cases may help empirically distinguish our proposal from that advanced by Gallistel and Gelman.

\section{Conclusion}

We have argued that the ANS represents numbers of a familiar sort, and tentatively suggested that this involves it representing both positive integers and rational numbers, but not the reals more generally. We have drawn this conclusion because arguments to the contrary are flawed (\$\$3-5), because the postulation of genuine number content has theoretical and explanatory advantages over the postulation of alternatives such as "quanticals," "pure magnitudes," or "numerosities" (\$6), and because the conjecture that these contents include natural and rational (but not irrational) numbers makes best sense of the existing data $(\$ 7)$. In so doing, we hope to have quelled recent skepticism surrounding the ANS's ability to represent number, clarified the nature of its representations, and highlighted fruitful questions to be investigated in future research.

\section{Acknowledgements}

We are extremely grateful to Greyson Abid, Elizabeth Brannon, Justin Halberda, Darko Odic, Kevin Lande, Nick Shea, Tom Walton, three anonymous referees, and the Editor of BBS for written suggestions on how to improve this manuscript; to Brian Ball, David Barner, Stephanie Denison, Brian Huss, Richard Murray, and Hans Robin Solberg for helpful discussions; to Andrew Buzzell for research assistance and helpful discussions; and to audiences at Boğaziçi University, Brown University, CUNY, UCSD, The University of Luxembourg, The University of Sheffield, The University of Toronto, York University, and the 2020 Meeting of the Cognitive Science Society. Both authors contributed equally to this manuscript.

\section{Funding Statement}

Both authors were supported in writing this paper by York University's Vision: Science to Applications Program, thanks in part to funding from the Canada First Research Excellence Fund. Jacob Beck was also supported by Social Sciences and Humanities Research Council of Canada Insight Grant 4352017-0923.

\section{Conflicts of Interest}

None.

\section{References}

1. Anobile, G., Arrighi, R., Castaldi, E., \& Burr, D. C. (2021). A Sensorimotor Numerosity System. Trends in Cognitive Sciences, 25(1), 24-36. https://doi.org/10.1016/j.tics.2020.10.009

2. Anobile, G., Cicchini, G. M., \& Burr, D. C. (2016). Number As a Primary Perceptual Attribute: A Review. Perception, 45(1-2), 5-31. https://doi.org/10.1177/0301006615602599

3. Arrighi, R., Togoli, I., \& Burr, D. C. (2014). A generalized sense of number. Proceedings of the Royal Society B: Biological Sciences, 281(1797), 20141791-20141791. https://doi.org/10.1098/rspb.2014.1791

4. Ball, B. (2017). On representational content and format in core numerical cognition. Philosophical Psychology, 30(1-2), 119 - 139. https://doi.org/10.1080/09515089.2016.1263988

5. Barth, H., Kanwisher, N., \& Spelke, E. (2003). The construction of large number representations in adults. Cognition, 86(3), 201-221. https://doi.org/10.1016/S0010-0277(02)00178-6

6. Barth, H., La Mont, K., Lipton, J., \& Spelke, E. S. (2005). Abstract number and arithmetic in preschool children. Proceedings of the National Academy of Sciences, 102(39), 1411614121. https://doi.org/10.1073/pnas.0505512102 
7. Barth, H., La Mont, K., Lipton, J., Dehaene, S., Kanwisher, N., \& Spelke, E. (2006). Non-symbolic arithmetic in adults and young children. Cognition, 98(3), 199222. https://doi.org/10.1016/j.cognition.2004.09.011

8. Barth, H., Baron, A., Spelke, E., \& Carey, S. (2009). Children's multiplicative transformations of discrete and continuous quantities. Journal of Experimental Child Psychology, 103(4), 441454. https://doi.org/10.1016/j.jecp.2009.01.014

9. Beck, J. (2013). Why we can't say what animals think. Philosophical Psychology, 26(4), 520-546. https://doi.org/10.1080/09515089.2012.670922

10. Beck, J. (2015). Analogue Magnitude Representations: A Philosophical Introduction. The British Journal for the Philosophy of Science, 66(4), 829-855. https://doi.org/10.1093/bjps/axu014

11. Beck, J. (2018). Analog mental representation. WIREs Cognitive Science, 9(6), e1479. doi.org/10.1002/wcs.1479

12. Beck, J. (2019). Perception is Analog: The Argument from Weber's Law. The Journal of Philosophy, 116(6), 319-349. https://doi.org/10.5840/jphil2019116621

13. Bhatia, P., Delem, M., Léone, J., et al. (2020). The ratio processing system and its role in fraction understanding: Evidence from a match-to-sample task in children and adults with and without dyscalculia. Quarterly Joumal of Experimental Psychology, 73(12): 2158-2176. doi:10.1177/1747021820940631

14. Binzak, J.V., \& Hubbard, E.M. (2020). No calculation necessary: Accessing magnitude through decimals and fractions. Cognition, 199: 104219. doi: 10.1016/j.cognition.2020.104219

15. Buijsman, S. (2021). The representations of the approximate number system. Philosophical Psychology, 34(2): 300-317. https://doi.org/10.1080/09515089.2020.1866755

16. Burge, T. (1982). Other bodies. In Andrew Woodfield (ed.), Thought and Object. Oxford University Press.

17. Burge, T. (2005). Truth, Thought, Reason: Essays on Frege. New York: Oxford University Press.

18. Burge, T. (2010). The origins of objectivity. Oxford: Oxford University Press.

19. Burr, D. \& Ross, J. (2008). A visual sense of number, Current Biology, 18(6), 425-8. doi: 10.1016/j.cub.2008.02.052.

20. Burr, D., Anobile, G., Togoli, I., Domenici, N., \& Arrighi, R. (2019). Motor adaptation affects perception of time and numerosity. Journal of Vision, 19(10), 164b164b. https://doi.org/10.1167/19.10.164b

21. Carey, S. (2009). The origin of concepts. Oxford: Oxford University Press.

22. Carey, S., \& Barner, D. (2020). Ontogenetic Origins of Human Integer Representations. Trends in Cognitive Sciences. https://doi.org/10.1016/j.tics.2019.07.004

23. Casasanto, D., \& Boroditsky, L. (2008). Time in the mind: Using space to think about time. Cognition, 106, 579-593. https://doi.org/10.1016/j.cognition.2007.03.004

24. Cheyette, S. J., \& Piantadosi, S. T. (2020). A unified account of numerosity perception. Nature Human Behaviour. https://doi.org/10.1038/s41562-020-00946-0

25. Church, R. M., \& Meck, W. H. (1983). The Numerical Attribute of Stimuli. Animal Cognition, 445.

26. Cicchini, G. M., Anobile, G., \& Burr, D. C. (2016). Spontaneous perception of numerosity in humans. Nature Communications, 7, 12536. https://doi.org/10.1038/ncomms12536

27. Clarke, S. (forthcoming a). Beyond the icon: Core cognition and the bounds of perception. Mind \& Language. https://doi.org/10.1111/mila.12315

28. Clarke, S. (forthcoming b). Cognitive Penetration and Informational Encapsulation: Have we been failing the module? Philosophical Studies. https://doi.org/10.1007/s11098-020-01565-1

29. Cohen Kadosh, R., \& Henik, A. (2006). A Common Representation for Semantic and Physical Properties. Experimental Psychology, 53(2), 87-94. https://doi.org/10.1027/1618-3169.53.2.87

30. Content, A. Velde, M., \& Adriano, A. (2017). Approximate number sense theory or approximate theory of magnitude? Behavioral and Brain Sciences, 40, E168. doi:10.1017/S0140525X16002089

31. Cordes, S., Gelman, R., Gallistel, R., \& Whalen, J. (2001). Variability signatures distinguish verbal from nonverbal counting for both large and small numbers. Psychonomic Bulletin \& Review, 8(4), 698707. 
32. Dakin, S. C., Tibber, M. S., Greenwood, J. A., Kingdom, F. A. A., \& Morgan, M. J. (2011). A common visual metric for approximate number and density. Proceedings of the National Academy of Sciences, 108(49), 19552-19557. https://doi.org/10.1073/pnas.1113195108

33. Dehaene, S. (2011). The Number Sense: How the Mind Creates Mathematics, Revised and Updated Edition: How the Mind Creates Mathematics. Oxford University Press, USA.

34. Dehaene S, Changeux JP. (1993). Development of elementary numerical abilities: a neuronal model. J Cogn Neurosci. 1993;5(4):390-407. doi:10.1162/jocn.1993.5.4.390

35. Dehaene, S., Izard, V., \& Piazza, M. (2005). Control over non-numerical parameters in numerosity experiments. http://www.unicog.org/docs/DocumentationDotsGeneration.doc $\% 3 \mathrm{E}$

36. Denison, S., \& Xu, F. (2010). Twelve- to 14-month-old infants can predict single-event probability with large set sizes. Developmental Science, 13(5), 798-803. https://doi.org/10.1111/j.14677687.2009.00943.x

37. Denison, S., \& Xu, F. (2014). The origins of probabilistic inference in human infants. Cognition, 130(3), 335-347. https://doi.org/10.1016/j.cognition.2013.12.001

38. DeSimone, K., Kim, M., \& Murray, R.F. (2020). Number Adaptation Can Be Dissociated From Density Adaptation. Psychological Science, 31(11),1470-1474. doi:10.1177/0956797620956986

39. DeWind, N. K., Adams, G. K., Platt, M. L., \& Brannon, E. M. (2015). Modeling the approximate number system to quantify the contribution of visual stimulus features. Cognition, 142, $247-$ 265. https://doi.org/10.1016/j.cognition.2015.05.016

40. Drucker, C. B., Rossa, M. A., \& Brannon, E. M. (2016). Comparison of discrete ratios by rhesus macaques (Macaca mulatta). Animal Cognition, 19(1), 75-89. https://doi.org/10.1007/s10071-0150914-9

41. Duhem, P. (1914). La théorie physique son objet et sa structure, 2nd ed., Paris: Chevalier et Rivière. English Translation Philip P. Wiener, The Aim and Structure of Physical Theory, Princeton: Princeton University Press, 1954.

42. Dummett, M. (1981). Frege: Pbilosophy of Language (London: Duckworth, and Cambridge MA: Harvard University Press, 2nd ed.)

43. Durgin FH. (2008). Texture density adaptation and visual number revisited, Current Biology, 18(18), R855-6. doi: 10.1016/j.cub.2008.07.053. PMID: 18812077.

44. Eckert, J., Call, J., Hermes, J., Herrmann, E., \& Rakoczy, H. (2018). Intuitive statistical inferences in chimpanzees and humans follow Weber's law. Cognition, 180, 99-107. https://doi.org/10.1016/j.cognition.2018.07.004

45. Evans, G. (1982). The V arieties of Reference. Oxford: Oxford University Press.

46. Feigenson, L., Dehaene, S., \& Spelke, E. (2004). Core systems of number. Trends in Cognitive Sciences, 8(7), 307-314. https://doi.org/10.1016/j.tics.2004.05.002

47. Fodor, J. (1983). The Modularity of Mind. Cambridge: MIT Press.

48. Fodor, J. (1998). Concepts: Where cognitive science went wrong. Oxford: OUP.

49. Fontanari, L., Gonzalez, M., Vallortigara, G., \& Girotto, V. (2014). Probabilistic cognition in two indigenous Mayan groups. Proceedings of the National Academy of Sciences, 111(48), 1707517080. https://doi.org/10.1073/pnas.1410583111

50. Fornaciai, M., Cicchini, G.M. \& Burr, D.C. (2016). Adaptation to number operates on perceived rather than physical numerosity, Cognition, 151, 63-67. https://doi.org/10.1016/j.cognition.2016.03.006

51. Fornaciai, M., \& Park, J. (2018). Early numerosity encoding in visual cortex is not sufficient for the representation of numerical magnitude. Journal of Cognitive Neuroscience, 30(12), 1788-1802. https://doi.org/10.1162/jocn_a_01320

52. Franconeri, S. L., Bemis, D. K., \& Alvarez, G. A. (2009). Number estimation relies on a set of segmented objects. Cognition, 113(1), 1-13. https://doi.org/10.1016/j.cognition.2009.07.002

53. Frege, G. (1884). Die Grundlagen der Arithmetik: eine logisch mathematische Untersuchung über den Begriff der Zabl, Breslau: W. Koebner; translated as The Foundations of Arithmetic: A logico-mathematical enquiry into the concept of number, by J.L. Austin, Oxford: Blackwell, second revised edition, 1974. 
54. Frege, G. (1892). Über Sinn und Bedeutung', in Zeitschrift für Philosophie und philosophische Kritik, 100: 25-50; translated as 'On Sense and Reference' by M. Black in Geach and Black (eds. and trans.), 1980, 56-78.

55. Gallistel, R., \& Gelman, R. (2000). Non-verbal numerical cognition: From reals to integers. Trends in Cognitive Sciences, 4(2), 59-65.

56. Gebuis T., Herfs I. K., Kenemans J. L., de Haan E. H. F., van der Smagt M. J. (2009). The development of automated access to number knowledge in children: an ERP study. Eur. J. Neurosci. 30, 1999-2008 10.1111/j.1460-9568.2009.06994.x

57. Gebuis, T., Kenemans, J. L., de Haan, E. H. F., \& van der Smagt, M. J. (2010). Conflict processing of symbolic and non-symbolic numerosity. Neuropsychologia, 48(2), 394 401. https://doi.org/10.1016/j.neuropsychologia.2009.09.027

58. Gebuis, T., Cohen Kadosh, R., \& Gevers, W. (2016). Sensory-integration system rather than approximate number system underlies numerosity processing: A critical review. Acta Psychologica, 171, 17-35. https://doi.org/10.1016/j.actpsy.2016.09.003

59. Gebuis, T., \& Reynvoet, B. (2012a). Continuous visual properties explain neural responses to nonsymbolic number. Psychophysiology, 49(11), 1649-1659. https://doi.org/10.1111/j.14698986.2012.01461.x

60. Gebuis, T., \& Reynvoet, B. (2012b). The interplay between nonsymbolic number and its continuous visual properties. Journal of Experimental Psychology: General, 141(4), 642 648. https://doi.org/10.1037/a0026218

61. Gebuis T, \& Reynvoet B (2012c) The Role of Visual Information in Numerosity Estimation. PLoS ONE, 7(5): e37426. https://doi.org/10.1371/journal.pone.0037426

62. Gergely G, Csibra G. (2003). Teleological reasoning in infancy: the nai;ve theory of rational action. Trends in Cognitive Sciences. 7(7):287-292. doi:10.1016/s1364-6613(03)00128-1

63. Girotto, V., \& Gonzalez, M. (2008). Children's understanding of posterior probability. Cognition, 106(1), 325-344. https://doi.org/10.1016/j.cognition.2007.02.005

64. Gordon, P. (2005). Numerical Cognition Without Words: Evidence from Amazonia. Science, 306(5695), 496-499. https://doi.org/10.1126/science.1094492

65. Green, E.J. (2018). Psychosemantics and the rich/thin debate. Philosophical Topics, 31(1), 153-86.

66. Greenwald, A. G., McGhee, D. E., \& Schwartz, J. L. K. (1998). Measuring individual differences in implicit cognition: The Implicit Association Test. Journal of Personality and Social Psychology, 74, 1464 1480. https://doi.org/10.1037/0022-3514.74.6.1464

67. Gweon, H., Tenenbaum, J. B., \& Schulz, L. E. (2010). Infants consider both the sample and the sampling process in inductive generalization. Proceedings of the National Academy of Sciences, 107(20), 9066-9071. https://doi.org/10.1073/pnas.1003095107

68. Halberda, J. (2016). Epistemic Limitations and Precise Estimates in Analog Magnitude Representation. In Oxford series in cognitive development. Core knowledge and conceptual change (pp. 171-190).

69. Halberda, J. (2019). Perceptual Input Is Not Conceptual Content. Trends in Cognitive Sciences. https://doi.org/10.1016/j.tics.2019.05.007

70. Halberda, J., Mazzocco, M. M. M., \& Feigenson, L. (2008). Individual differences in non-verbal number acuity correlate with maths achievement. Nature, 455(7213), 665668. https://doi.org/10.1038/nature07246

71. He, L., Zhang, J., Zhou, T., \& Chen, L. (2009). Connectedness affects dot numerosity judgment: Implications for configural processing. Psychonomic Bulletin \& Review, 16(3), 509517. https://doi.org/10.3758/PBR.16.3.509

72. Henik, A., \& Tzelgov, J. (1982). Is three greater than five: The relation between physical and semantic size in comparison tasks. Memory \& Cognition, 10(4), 389-395. https://doi.org/10.3758/BF03202431

73. Irie, N., Hiraiwa-Hasegawa, M., \& Kutsukake, N. (2019). Unique numerical competence of Asian elephants on the relative numerosity judgment task. Journal of Ethology, 37(1), 111115. https://doi.org/10.1007/s10164-018-0563-y 
74. Izard, V., Sann, C., Spelke, E. S., \& Streri, A. (2009). Newborn infants perceive abstract numbers. Proceedings of the National Academy of Sciences, 106(25), 10382-

10385. https://doi.org/10.1073/pnas.0812142106

75. Johnson, A (2004). Attention: theory and practice. Thousand Oaks, Calif: Sage Publications.

76. Kayhan, E., Gredebäck, G., \& Lindskog, M. (2018). Infants Distinguish Between Two Events Based on Their Relative Likelihood. Child Development, 89(6), e507e519. https://doi.org/10.1111/cdev.12970

77. Kirjakovski, A., \& Matsumoto, E. (2016). Numerosity underestimation in sets with illusory contours. Vision Research, 122, 34 42. https://doi.org/10.1016/j.visres.2016.03.005

78. Kominsky, J. \& Carey, S. (2018). Early-Developing Causal Perception is Sensitive to Multiple Physical Constraints. Proceedings of the Cognitive Science Society (41). https://cogsci.mindmodeling.org/2018/papers/0134/index.html

79. Laurence, S., \& Margolis, E. (2005). Number and Natural. The Innate Mind: Structure and Contents, 1 , 216.

80. Leibovich, T., \& Henik, A. (2013). Magnitude processing in non-symbolic stimuli. Frontiers in Psychology, 4. https://doi.org/10.3389/fpsyg.2013.00375

81. Leibovich, T., \& Henik, A. (2014). Comparing Performance in Discrete and Continuous Comparison Tasks. Quarterly Journal of Experimental Psychology, 67(5), 899-

917. https://doi.org/10.1080/17470218.2013.837940

82. Leibovich, T., Katzin, N., Harel, M., \& Henik, A. (2017). From 'sense of number' to 'sense of magnitude' - The role of continuous magnitudes in numerical cognition. Behavioral and Brain Sciences, 1-62. https://doi.org/10.1017/S0140525X16000960

83. Lemer, C., Dehaene, S., Spelke, E., \& Cohen, L. (2003). Approximate quantities and exact number words: Dissociable systems. Neuropsychologia, 41(14), 1942-1958. https://doi.org/10.1016/S00283932(03)00123-4

84. Lewis, D. (1971). Analog and digital. Noûs, 5(3), 321-27. doi: 10.2307/2214671

85. Lewis, M. R., Matthews, P. G., \& Hubbard, E. M. (2016). Chapter 6-Neurocognitive Architectures and the Nonsymbolic Foundations of Fractions Understanding. In D. B. Berch, D. C. Geary, \& K. M. Koepke (Eds.), Development of Mathematical Cognition (pp. 141-164). Academic Press. https://doi.org/10.1016/B978-0-12-801871-2.00006-X

86. Lipton, J.S. \& Spelke, E.S. (2003). Origins of Number Sense: Large-Number Discrimination in Human Infants. Psychological Science, 14(5), 396-401.

87. Lourenco, S. F., \& Longo, M. R. (2010). General Magnitude Representation in Human Infants. Psychological Science, 21(6), 873-881. https://doi.org/10.1177/0956797610370158

88. Lucero, C., Brookshire, G., Sava-Segal, C. Bottini, R., Goldin-Meadow, S., Vogel, E., \& Casasanto, D. (2020). Unconscious number discrimination in the human visual system. Cerebral Cortex. DOI:10.1093/cercor/bhaa155

89. Maley, C. J. (2011). Analog and digital, continuous and discrete. Philosophical Studies, 155(1), 117 131. https://doi.org/10.1007/s11098-010-9562-8

90. Mandelbaum, E. (2013). Numerical Architecture. Topics in Cognitive Science, 5(2), 367386. https://doi.org/10.1111/tops.12014

91. Margolis, E. (2020). The Small Number System. Pbilosopby of Science, 87(1), 113134. https://doi.org/10.1086/706087

92. Margolis, E., \& Laurence, S. (2008). How to learn the natural numbers: Inductive inference and the acquisition of number concepts. Cognition, 106(2), 924939. https://doi.org/10.1016/j.cognition.2007.03.003

93. Marr, D. (1982). Vision: a computational investigation into the buman representation and processing of visual information. Cambridge, MA: MIT Press.

94. Marshall, O. R. (2017). The Psychology and Philosophy of Natural Numbers. Philosophia Mathematica, 26(1), 40-58. https://doi.org/10.1093/philmat/nkx002

95. Martin, B., Wiener, M., \& van Wassenhove, V. (2017). A Bayesian Perspective on Accumulation in the Magnitude System. Scientific Reports, 7(1), 630. https://doi.org/10.1038/s41598-017-00680-0 
96. Matthews, P. G., \& Chesney, D. L. (2015). Fractions as percepts? Exploring cross-format distance effects for fractional magnitudes. Cognitive Psychology, 78, 28-

56. https://doi.org/10.1016/j.cogpsych.2015.01.006

97. Matthews PG, Lewis MR, Hubbard EM. (2016). Individual Differences in Nonsymbolic Ratio

Processing Predict Symbolic Math Performance. Psychological Science, 27(2):191-202. doi:10.1177/0956797615617799

98. McCrink, K., \& Wynn, K. (2007). Ratio Abstraction by 6-Month-Old Infants. Psychological Science, 18(8), 740-745. https://doi.org/10.1111/j.1467-9280.2007.01969.x

99. McCrink, K., \& Spelke, E. S. (2010). Core multiplication in childhood. Cognition, 116(2), 204 216. https://doi.org/10.1016/j.cognition.2010.05.003

100.McCrink, K., \& Spelke, E. S. (2016). Non-symbolic division in childhood. Journal of Experimental Child Psychology, 142, 66-82. https://doi.org/10.1016/j.jecp.2015.09.015

101.McCrink, K., Spelke, E. S., Dehaene, S., \& Pica, P. (2012). Non-symbolic halving in an Amazonian indigene group. Developmental Science, 16(3), 451-462. https://doi.org/10.1111/desc.12037

102.Morgan, M. J., Raphael, S., Tibber, M. S., \& Dakin, S. C. (2014). A texture-processing model of the 'visual sense of number.' Proceedings of the Royal Society B: Biological Sciences, 281(1790), 20141137. https://doi.org/10.1098/rspb.2014.1137

103.Neander, K. (2017). A Mark of the Mental: A Defence of Informational Teleosemantics. Cambridge, USA: MIT Press.

104.Nieder, A. (2016). The neuronal code for number. Nature Reviews Neuroscience, 17(6), $366-$ 382. https://doi.org/10.1038/nrn.2016.40

105.Nieder, A. (2017). Number Faculty Is Rooted in Our Biological Heritage. Trends in Cognitive Sciences, 21(6), 403-404. https://doi.org/10.1016/j.tics.2017.03.014

106.Nieder, A. (2020). Neural constraints on human number concepts. Current Opinion in Neurobiology, 60, 28-36. https://doi.org/10.1016/j.conb.2019.10.003

107.Núñez, R. E. (2017). Is There Really an Evolved Capacity for Number? Trends in Cognitive Sciences, 21(6), 409-424. https://doi.org/10.1016/j.tics.2017.03.005

108.Nys, J., \& Content, A. (2012). Judgement of discrete and continuous quantity in adults: Number counts! The Quarterly Journal of Experimental Psychology, 65(4), 675690. https://doi.org/10.1080/17470218.2011.619661

109. Odic, D. (2018). Children's intuitive sense of number develops independently of their perception of area, density, length, and time. Developmental Science, 21(2), e12533. https://doi.org/10.1111/desc.12533

110.Odic, D., \& Starr, A. (2018). An Introduction to the Approximate Number System. Child Development Perspectives, 12(4), 223-229. https://doi.org/10.1111/cdep.12288

111.Park, J., \& Brannon, E. M. (2013). Training the Approximate Number System Improves Math Proficiency. Psychological Science, 24(10), 2013-2019. https://doi.org/10.1177/0956797613482944

112.Park, J., DeWind, N, \& Brannon, E. (2017). Direct and rapid encoding of numerosity in the visual stream. Behavioral and Brain Sciences, 40, E185. Doi:10.1017/S0140525X16002235.

113.Peacocke, C. (1986). Analogue content. Proceedings of the Aristotelian Society, Supplementary volumes, 601 17. https://www.jstor.org/stable/4106896

114.Peacocke, C. (1992). A Study of Concepts. Cambridge, MA: MIT Press.

115.Peacocke, C. (2020). The Primacy of Metaphysics. Oxford: OUP.

116.Petzschner, F. H., Glasauer, S., \& Stephan, K. E. (2015). A Bayesian perspective on magnitude estimation. Trends in Cognitive Sciences, 19(5), 285-293. https://doi.org/10.1016/j.tics.2015.03.002

117.Piazza, M. (2011). Neurocognitive start-up tools for symbolic number representations. Trends in Cognitive Sciences, 14(12), 542-551. https://doi.org/10.1016/j.tics.2010.09.008

118.Pica, P., Lemer, C., Izard, V., \& Dehaene. S. (2005). Exact and Approximate Arithmetic in an Amazonian Indigene Group. Science, 306(5695), 499-503. https://doi.org/10.1126/science.1102085

119.Picon, E., Dramkin, D., \& Odic, D. (2019). Visual illusions help reveal the primitives of number perception. Journal of Experimental Psychology: General, 148(10), 16751687. https://doi.org/10.1037/xge0000553 
120.Plotnik, J. M., Brubaker, D. L., Dale, R., Tiller, L. N., Mumby, H. S., \& Clayton, N. S. (2019). Elephants have a nose for quantity. Proceedings of the National Academy of Sciences, 116(25), 1256612571. https://doi.org/10.1073/pnas.1818284116

121.Putnam, Hilary (1975). The meaning of 'meaning'. Minnesota Studies in the Pbilosophy of Science 7:131193. http://hdl.handle.net/11299/185225

122.Quine, W.V. (1951). Two dogmas of empiricism, Philosophical Review, 60, 20-43.

123.Rakoczy, H., Clüver, A., Saucke, L., Stoffregen, N., Gräbener, A., Migura, J., \& Call, J. (2014). Apes are intuitive statisticians. Cognition, 131(1), 60-68. https://doi.org/10.1016/j.cognition.2013.12.011

124.Sarrazin, J.-C., Giraudo, M.-D., Pailhous, J., \& Bootsma, R. J. (2004). Dynamics of Balancing Space and Time in Memory: Tau and Kappa Effects Revisited. Journal of Experimental Psychology: Human Perception and Performance, 30(3), 411-430. https://doi.org/10.1037/0096-1523.30.3.411

125.Savelkouls, S., \& Cordes, S. (2020). The impact of set size on cumulative area judgments. Acta Psychologica, 210, 103163. https://doi.org/10.1016/j.actpsy.2020.103163

126.Shea, Nicholas (2018). Representation in Cognitive Science. Oxford: Oxford University Press.

127.Siegler, R. S., Fazio, L. K., Bailey, D. H., \& Zhou, X. (2013). Fractions: The new frontier for theories of numerical development. Trends in Cognitive Sciences, 17(1), 13-19. doi: 10.1016/j.tics.2012.11.004

128.Skudlarek, E. \& Brannon, E. (2021). First and Second Graders Successfully Reason About Ratio With Both Dot Arrays and Arabic Numerals. Child Development. 1-17.

129.Starr, A., Libertus, M. E., \& Brannon, E. M. (2013). Number sense in infancy predicts mathematical abilities in childhood. Proceedings of the National Academy of Sciences, 110(45), 1811618120. https://doi.org/10.1073/pnas.1302751110

130. Tecwyn, E. C., Denison, S., Messer, E. J. E., \& Buchsbaum, D. (2017). Intuitive probabilistic inference in capuchin monkeys. Animal Cognition, 20(2), 243-256. https://doi.org/10.1007/s10071016-1043-9

131.Téglás, E., Vul, E., Girotto, V., Gonzalez, M., Tenenbaum, J. B., \& Bonatti, L. L. (2011). Pure Reasoning in 12-Month-Old Infants as Probabilistic Inference. Science, 332(6033), 1054 1059. https:// doi.org/10.1126/science. 1196404

132.Tenenbaum, J. B., Kemp, C., Griffiths, T. L., \& Goodman, N. D. (2011). How to Grow a Mind: Statistics, Structure, and Abstraction. Science, 331(6022), 12791285. https://doi.org/10.1126/science. 1192788

133.Tokita, M., Ishiguchi, A. (2012). Behavioral evidence for format-dependent processes in approximate numerosity representation. Psychon Bull Rev 19, 285-293. https://doi.org/10.3758/s13423-011-0206-6

134.Tokita, M., Ashitani, Y., \& Ishiguchi, A. (2013). Is approximate numerical judgment truly modalityindependent? Visual, auditory, and cross-modal comparisons. Attention, Perception, \& Psychophysics, 75(8), 1852-1861. https://doi.org/10.3758/s13414-013-0526-x

135.Tomlinson, R. C., DeWind, N. K., \& Brannon, E. M. (2020). Number sense biases children's area judgments. Cognition, 204, 104352. https://doi.org/10.1016/j.cognition.2020.104352

136.von Neumann, J. (1958). The Computer and the Brain

137.Wagner, J. B., \& Johnson, S. C. (2011). An association between understanding cardinality and analog magnitude representations in preschoolers. Cognition, 119(1), 1022. https://doi.org/10.1016/j.cognition.2010.11.014

138.Wood, J. N., \& Spelke, E. S. (2005). Infants' enumeration of actions: Numerical discrimination and its signature limits. Developmental Science, 8(2), 173-181. https://doi.org/10.1111/j.14677687.2005.00404.x

139.Xu, F., \& Denison, S. (2009). Statistical inference and sensitivity to sampling in 11-month-old infants. Cognition, 112(1), 97-104. https://doi.org/10.1016/j.cognition.2009.04.006

140.Xu, F., \& Garcia, V. (2008). Intuitive statistics by 8-month-old infants. Proceedings of the National Academy of Sciences, 105(13), 5012-5015. https://doi.org/10.1073/pnas.0704450105

141.Xu, F., \& Spelke, E. S. (2000). Large number discrimination in 6-month-old infants. Cognition, 74(1), B1-B11. https://doi.org/10.1016/S0010-0277(99)00066-9

142.Xuan, B., Zhang, D., He, S., Chen, X. (2007). Larger stimuli are judged to last longer. Journal of Vision, $7(10): 2,1-5$. https://doi.org/10.1167/7.10.2 
143.Yousif, S. R., \& Keil, F. C. (2020). Area, not number, dominates estimates of visual quantities. Scientific Reports, 10(1), 13407. https://doi.org/10.1038/s41598-020-68593-z

144.Zimmermann, E. (2018). Small numbers are sensed directly, high numbers constructed from size and density. Cognition, 173, 1-7. https://doi.org/10.1016/j.cognition.2017.12.003 